11 Abstract: Alloying anodes have long been attracting attention as promising candidate electrodes

12 for application in grid-level energy storage systems owing to their high energy capacity. Alloying

13 anode-based batteries, however, remain far from practical applications, which require several

14 issues affecting cell performance to be addressed. The large volumetric expansion of anodes and

\section{Extending the performance limit of anodes: insights from diffusion} kinetics of alloying anodes \author{
Yong-Seok Choi, ${ }^{1,2,3}$ David O. Scanlon, ${ }^{1,2,3,4}$ and Jae-Chul Lee ${ }^{5, *}$ \\ ${ }^{1}$ Department of Chemistry, University College London, 20 Gordon Street, London WC1H 0AJ, UK \\ ${ }^{2}$ Thomas Young Centre, University College London, Gower Street, London WC1E 6BT, UK \\ ${ }^{3}$ The Faraday Institution, Harwell Campus, Didcot OX11 0RA, UK \\ ${ }^{4}$ Diamond Light Source Ltd., Diamond House, Harwell Science and Innovation Campus, Didcot, \\ ${ }^{5}$ Department of Materials Science and Engineering, Korea University, Seoul, 02841, South Korea \\ Oxfordshire OX11 0DE, UK
}


23 electrochemical performance of alloying anodes is discussed, combined with efficient strategies

24 that can be adopted to improve electrochemical properties. Finally, we propose a design overview 25 of next-generation alloying anodes that can extend the batteries' performance limit.

26

27 Keywords: rechargeable battery, alloying anodes, diffusion kinetics, anisotropic swelling, self28 limiting diffusion.

29

30 *To whom correspondence should be addressed. Jae-Chul Lee (email: jclee001@korea.ac.kr)

31 


\section{Introduction}

Since the first commercialization of lithium-ion batteries (LIBs) in the early $1990{ }^{[1]}$, LIBs,

34 in conjunction with transition metal oxide cathodes ${ }^{[2,3]}$ and carbonaceous anodes, have been

35 attracting considerable attention as advanced energy storage devices ${ }^{[4]}$. The current success of

36 LIBs is primarily attributed to the insertion mechanism of Li ions that occurs between the layers

37 of host electrodes ${ }^{[5,6]}$. This mechanism allows electrodes to operate without significant structural

38 changes for prolonged periods and enables LIBs to exhibit a long cycle life, high stability, and

39 moderate energy capacity that are close to their theoretical limits (Figure 1) ${ }^{[3,7-16]}$. However, with

40 the rapid expansion in the capacity demands of electric vehicles and grid-scale energy storage

41 systems, current electrodes based on the insertion mechanism are being criticized for their limited

42 energy density.

In a continuous effort by the research community to develop high-performance

44 rechargeable batteries, electrode materials that follow alternative mechanisms have been

45 investigated ${ }^{[17]}$. Electrochemical processes involving conversion and alloying mechanisms exhibit

46 much greater theoretical capacities than those of processes based on the insertion mechanism, thus

47 enabling the realization of high-energy-density electrodes for next-generation batteries (Figure 1).

48 The high theoretical capacity of conversion and alloying electrodes originates from their unique

49 uptake mechanisms of carrier ions; unlike insertion electrodes, conversion and alloying electrodes

50 accommodate carrier ions by disrupting the crystal structure of host electrodes. This allows a large

51 number of carrier ions to be stored without the restraint of the crystal frameworks of electrodes,

52 which is particularly true for alloying anodes; $\mathrm{Si}$, for example, can accept Li ions up to 4.4 per unit

53 formula and yield a theoretical capacity of $4200 \mathrm{mAhg}^{-1}$ (Figure 1a). However, the alloying of Si

54 with Li inevitably causes large volumetric changes in the Si anodes during electrochemical cycling 
$55[18,19]$, resulting in performance issues associated with the mechanical degradation of $\mathrm{Si}$, such as a 56 short cycling life and low practical capacity.

57 The increasing knowledge gap between the degradation process and changes in the 58 electrochemical properties of alloying anodes has motivated various theories and experiments on 59 the diffusion kinetics of alloying anodes ${ }^{[11-15,17]}$. Accumulated models and experimental evidence 60 reveal that carrier ions are diffused into alloying anodes by forming an intermediate reaction layer 61 that separates the unreacted alloying anodes from inflowing carrier ions. This diffusion process, 62 often referred to as two-phase reaction/diffusion, means that the overall diffusion rate is governed 63 either by the diffusion rate of charge carriers at the propagating phase boundary $\left(v_{i}\right)$ or that at bulk 64 regions $\left(v_{b}\right)$ of the trailing intermediate reaction layer (Figures $\left.1 \mathrm{~b}\right)^{[20]}$. The former mode of 65 diffusion is termed an interface-controlled reaction (ICR), whereas the latter is called a diffusion66 controlled reaction (DCR) ${ }^{[21]}$. Although various electrochemical properties of alloying anodes are 67 closely related to their diffusion kinetics, their relationship remains largely unexplained because 68 of a lack of appropriate characterization of the atomic structures of the materials present. In the 69 past decade, there has been significant progress in nanotechnology and atomic simulations that has 70 enabled exploring the diffusional behaviors (e.g., orientation-dependent diffusion, diffusion rate, 71 and penetration depth of carrier ions) in alloying anodes and their effect on anode performance. In 72 this regard, an in-depth understanding of the diffusion kinetics of alloying anodes can offer a 73 rational strategy for the design and architecture of advanced anode materials with enhanced 74 properties.

This progress report, mainly from the perspective of diffusion kinetics, aims to understand 76 the origin of the electrochemical behaviors of alloying anodes and address their poor 77 electrochemical performance (i.e., cycle life, rate performance, and practical capacity). First, we 
78 summarize the research history of diffusion kinetics and its extension to alloying anodes.

79 Additionally, three sections successively discuss details on the electrochemical performance of

80 alloying anodes and their relationship with diffusion kinetics. Finally, on the basis of this

81 relationship, we clarify two important material properties, i.e., bond strength and yield strength,

82 for determining anode performance, which can be used as material design principles for

83 developing future batteries with superior electrochemical performance.
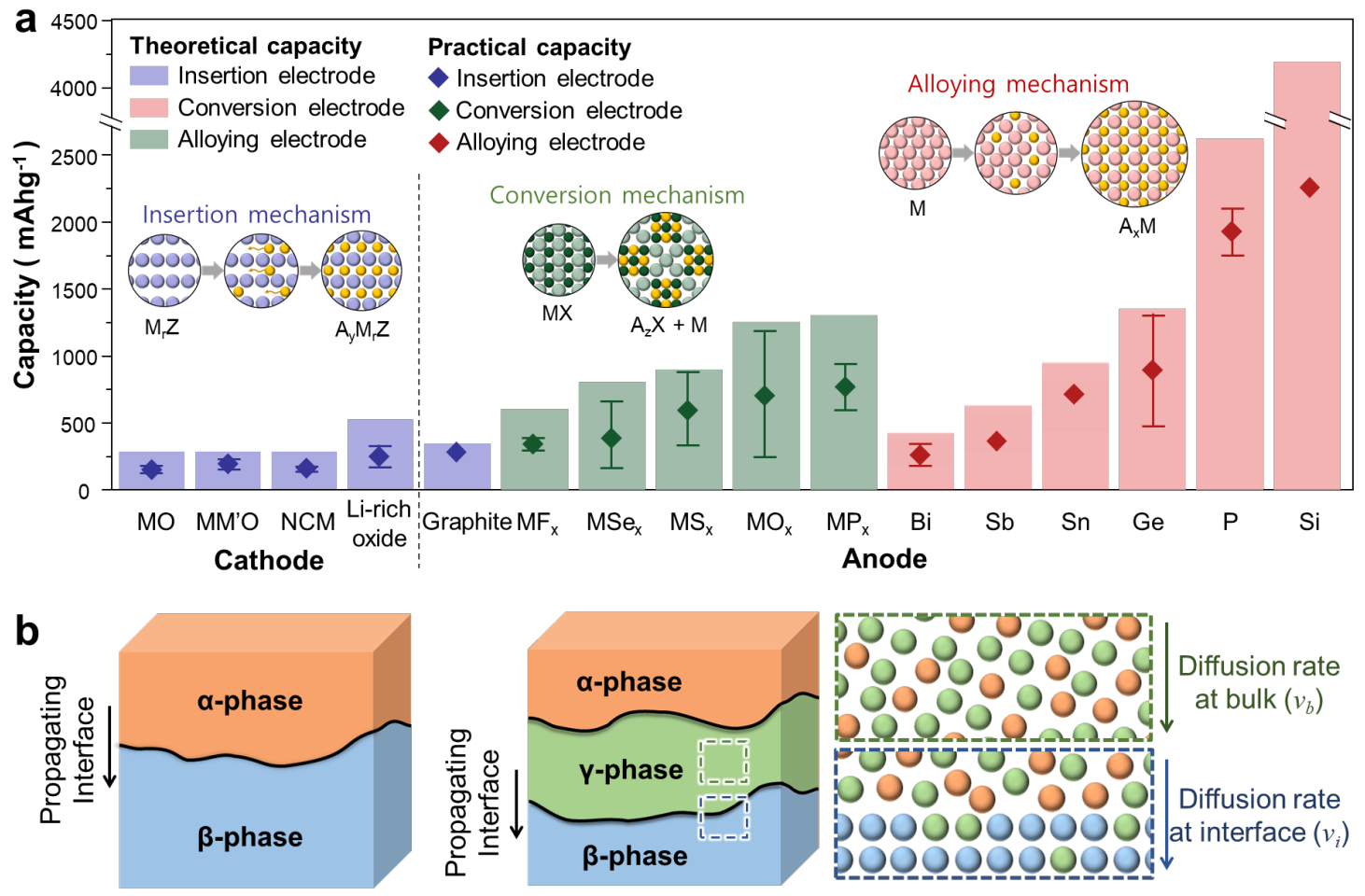

86 Figure 1. (a) Comparison of the theoretical (colored bars) and practical ( $\bullet$ ) capacities of cathode $87{ }^{[3,7,8]}$ and anode ${ }^{[9-16]}$ materials governed by different diffusion mechanisms. The practical capacities

88 are obtained by averaging the specific capacities of Li half cells measured after 20 cycles. The 89 error bars indicate the maximum and minimum values of the capacities reported for each electrode.

90 (b) Schematic of a diffusion couple showing that the diffusion in alloying anodes proceeds by 91 forming an intermediate reaction layer that allows the diffusion to occur via the two-phase reaction.

\section{2. Research history of diffusion kinetics}




\subsection{Early studies on diffusion kinetics}

Studies on diffusion kinetics were initially triggered by the need to control the oxidation

96 behavior of metals. An oxide layer, when growing slowly by adhering to the metal surface, can act

97 as a passive layer that can protect the metal from further oxidation. Conversely, loose, fast-growing

98 oxide layers tend to degrade substrate metals, causing them to fail after prolonged use. For 99 engineering design, therefore, an understanding of the oxidation rate is an important task in 100 estimating the lifespan of metals, and this requirement motivated various experiments aiming to 101 measure the oxidation rate of metals in the early 1920s. Based on accumulated experimental data, 102 Wagner established the diffusion kinetics by relating the oxide layer thickness $(L)$ and oxidation 103 time $(t)$ by the $L^{2} \propto t$ law (also called the parabolic rate law and later termed DCR) ${ }^{[22,23]}$. This 104 parabolic relation is obtained because the oxidation rate at the propagating phase boundary (or 105 interface) is faster than that in trailing bulk oxide regions; consequently, the overall diffusion rate 106 is governed by the reactions in the trailing bulk regions. In addition to the parabolic rate law, the 107 linear rate law of $L \propto t$ occurs when the diffusion rate of solute atoms is faster at the trailing 108 reaction layer than that at the propagating phase boundary or interface. This linear relation is 109 obtained because the overall diffusion rate is limited by the reactions at the interface, which are 110 called ICR ${ }^{[24,25]}$.

111 The concepts of ICR and DCR were actively discussed for conventional alloys in the late 112 20th century to explain the relationship between the diffusion kinetics and the reactions that limit 113 the overall diffusion rate in conventional alloys. Experiments reported in this period ${ }^{[26-28]}$ were 114 mainly on DCR diffusion (Figure 2a) and analyzed using various numerical models for solid-state 115 diffusion. Large-scale simulation based on finite element method (FEM) analysis is probably the 116 most common method used to analyze the local compositions and respective stress states under a 
117 given diffusion kinetics. Murray et al. developed a generic computational model for the solid-state

118 diffusion of systems governed by DCR ${ }^{[29]}$. This finite element (FE) model assumes the local

119 equilibrium state at the phase boundary and allows the prediction of the position of the moving 120 phase boundary as a function of time; this model was later used as a basis for calculating local 121 stresses near the propagating interface ${ }^{[30]}$.

122 The DCR diffusion model was subsequently extended to ICR diffusion by considering the 123 non-equilibrium condition at the phase boundary; then, it was used to evaluate various parameters 124 affecting diffusion behaviors ${ }^{[31]}$. In the 2000s, with the development of advanced analytical 125 instruments, the direct observation of moving phase boundaries became feasible, enabling detailed 126 studies on the diffusion kinetics and associated changes in microstructures. In-situ experiments 127 performed to measure the thickness of the reaction layer at the interface confirmed that the $L \propto t$ 128 law and orientation-dependent diffusion are valid for systems governed by ICR (Figure 2b) ${ }^{[32,33] .}$ 129 Based on these observations, Jeffrey et al. speculated that ICR can be responsible for the 130 anisotropic diffusion, often observed in the Li-Si system ${ }^{[34]}$.
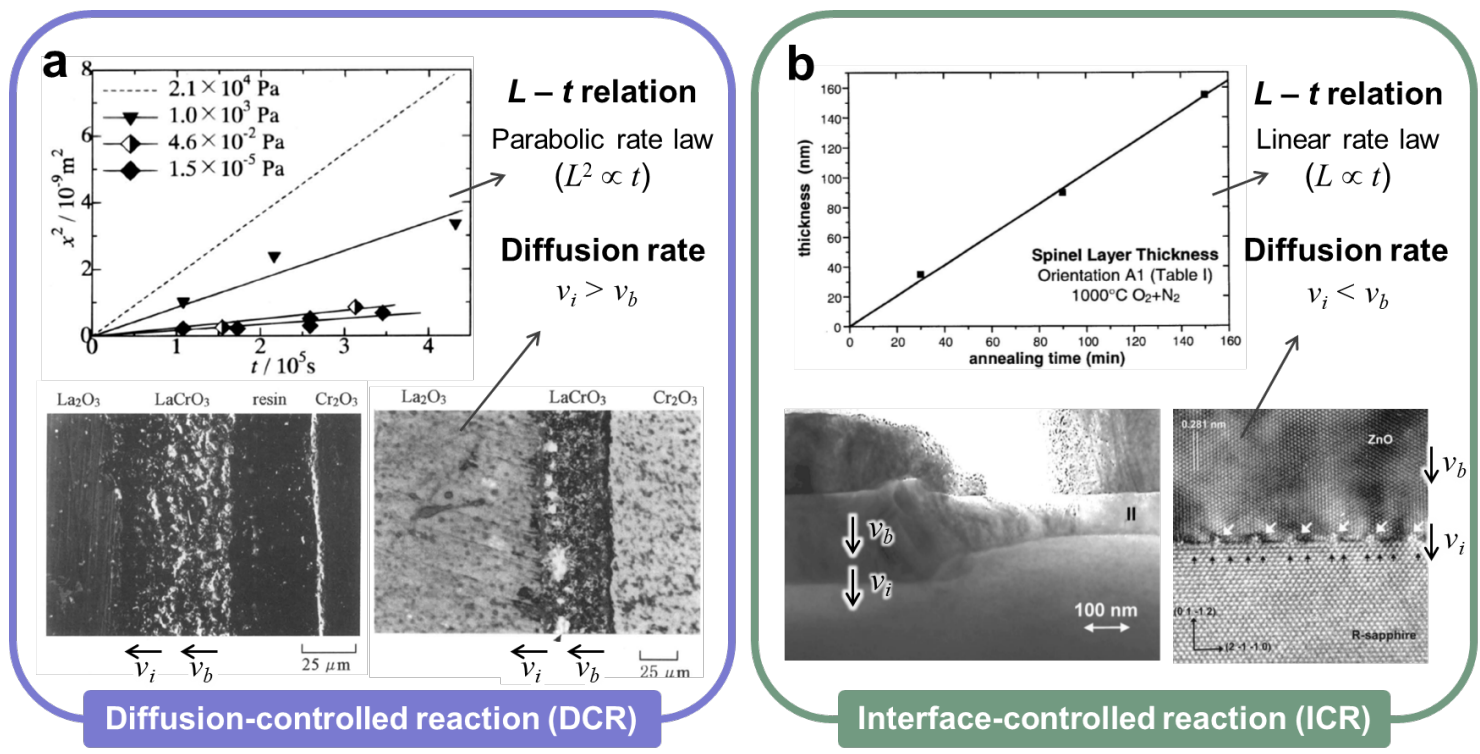
133 Figure 2. Representative works on the diffusion behaviors governed by (a) DCR ${ }^{[35]}$ and (b) ICR

$134{ }^{[32]}$. The systems governed by DCR and ICR are characterized by the $L^{2} \propto t$ and $L \propto t$ laws, 135 respectively. Reproduced with permission from Refs. ${ }^{[32,35]}$ Copyright (C 1998 IOP publishing and 136 C 2000 AIP publishing LLC.

\subsection{Extension of theories on diffusion kinetics to alloying anodes}

Although theories on diffusion kinetics were introduced for conventional alloys in the early

140 1920s, it was not until the 2010s when researchers began to adopt these theories to explore various

141 diffusion behaviors occurring in alloying anodes. Huang et al. developed the first open-cell micro-

142 electrochemical device that can be mounted inside an in-situ transmission electron microscopy

143 (TEM) instrument ${ }^{[36]}$ and observed the microstructural evolution of Si nanowires (NWs) during

144 Li diffusion ${ }^{[19]}$. They observed that the Li diffusion in Si NWs proceeds by forming an amorphous

$145 \mathrm{Li}_{x} \mathrm{Si}$ layer, thereby establishing two-phase diffusion. Experiments show that the propagation

146 length $(L)$ of the interface is linearly related to the diffusion time $(t)$, suggesting that the lithiation

147 of crystalline $\mathrm{Si}$ is governed by ICR. This $L \propto t$ relation arises from short-range diffusion occurring

148 at atomically sharp interfaces with a thickness of $\sim 1 \mathrm{~nm}^{[18]}$. This suggests that the Li diffusion at

149 the interface is too slow to transport Li ions to regions away from the interface, causing the entire

150 diffusion to be governed by ICR. Furthermore, the short-range diffusion occurring at the interface

151 causes the Li diffusion rate to differ depending on the orientation of the crystalline $\mathrm{Si}$, rendering

152 the volume change of the lithiated Si NWs highly anisotropic ${ }^{[37]}$.

153 Because lithiation behaviors are directly influenced by the nature of the $\mathrm{Si} / \mathrm{Li}_{x} \mathrm{Si}$ interface, 154 model studies were performed to evaluate the physics underlying the lithiation of Si. In early model 155 studies on the Li/Si diffusion couple, researchers commonly assumed the lithiation rate of Si to be 
156 limited by the diffusion rate at the trailing $\mathrm{Li}_{x} \mathrm{Si}$ region (i.e., DCR), while ignoring the orientation-

157 dependent diffusion of the advancing interface ${ }^{[38,39]}$ and thus failed to reproduce the sharp $\mathrm{Si} / \mathrm{Li}_{x} \mathrm{Si}$

158 interface and abrupt changes in the Li composition observed in experiments. To predict the abrupt

159 changes in the composition and the associated development of residual stresses at the $\mathrm{Si} / \mathrm{Li}_{x} \mathrm{Si}$

160 interface, new models were created by assuming the lithiation rate of Si NWs to be limited by ICR

161 diffusion. Models based on ICR diffusion could not only explain the crack formation behaviors of

162 Si particles during lithiation ${ }^{[40]}$ but also reproduce anisotropic morphologies of lithiated Si NWs

$163{ }^{[37]}$. Another important finding observed from Li/Si systems is that ICR diffusion is influenced by

164 various material characteristics, such as the molar volume of $\mathrm{Si}$, Li diffusivity in $\mathrm{Li}_{x} \mathrm{Si}$, radius of

$165 \mathrm{Si}$ particles, and rate constants for the reaction toward $\mathrm{Si}{ }^{[20]}$. These results obtained from $\mathrm{Li} / \mathrm{Si}$

166 systems explain why Si anodes exhibit abrupt changes in the Li composition profiles ${ }^{[37,40]}$, high

167 residual stresses ${ }^{[20]}$, anisotropic swelling ${ }^{[37]}$, and associated crack formation ${ }^{[40]}$, which are closely

168 related to such anodes' electrochemical performance.

169 Studies on alloying anodes governed by DCR diffusion began only in the late 2010 s.

170 Ironically, the first reported case of DCR diffusion is also the lithiation of crystalline Si. Seo at al.

171 performed direct-contact lithiation experiments using Si NWs with a diameter of $\sim 80 \mathrm{~nm}{ }^{[41]}$. They

172 observed that the propagation length $(L)$ of the reaction front changes with time $(t)$ according to

173 the $L^{2} \propto t$ relation, indicating that the lithiation of the 80-nm-thick Si NWs follows DCR. On the

174 other hand, the lithiation of Si NWs with diameters greater than $100 \mathrm{~nm}$ follows ICR ${ }^{[19,42]}$. The

175 contradictory results obtained from the above experiments indicate that the diameter/size of anode

176 materials is another parameter affecting the diffusion kinetics of alloying anodes. Notably, the

177 transition in diffusion kinetics from ICR to DCR significantly improves the Li diffusion rate in Si

178 NWs; the propagation speed of the lithiation front measured from the 80-nm-diameter Si NWs was 
$1791082 \mathrm{~nm} \mathrm{~s}^{-1}{ }^{[41]}$, which is nearly three orders of magnitude greater than that measured for 140-nm-

180 diameter Si NWs $\left(1.7 \mathrm{~nm} \mathrm{~s}^{-1}\right)^{[43]}$. In addition to Li/Si systems, DCR diffusion was observed to

181 occur in $\mathrm{Na} / \mathrm{Sn}{ }^{[44]}, \mathrm{Li} / \mathrm{Ge}^{[45]}$, and $\mathrm{Na} / \mathrm{Ge}^{[46]}$ systems. Contrary to systems governed by ICR,

182 systems following DCR diffusion commonly display 1) comparatively fast diffusion rates, 2)

183 isotropic swelling or expansion, and 3) deep penetration depths of carrier ions. All these behaviors

184 are desirable for improving the electrochemical properties of alloying anodes, as typically revealed

185 by charge rate, cycle life, and energy capacity. Therefore, diffusion kinetics and its effects on

186 anode performance should be understood to extend/break the performance limit of conventional

187 alloying anodes. This point is elaborated in the following section by relating diffusion kinetics

188 with the abovementioned diffusion behaviors.

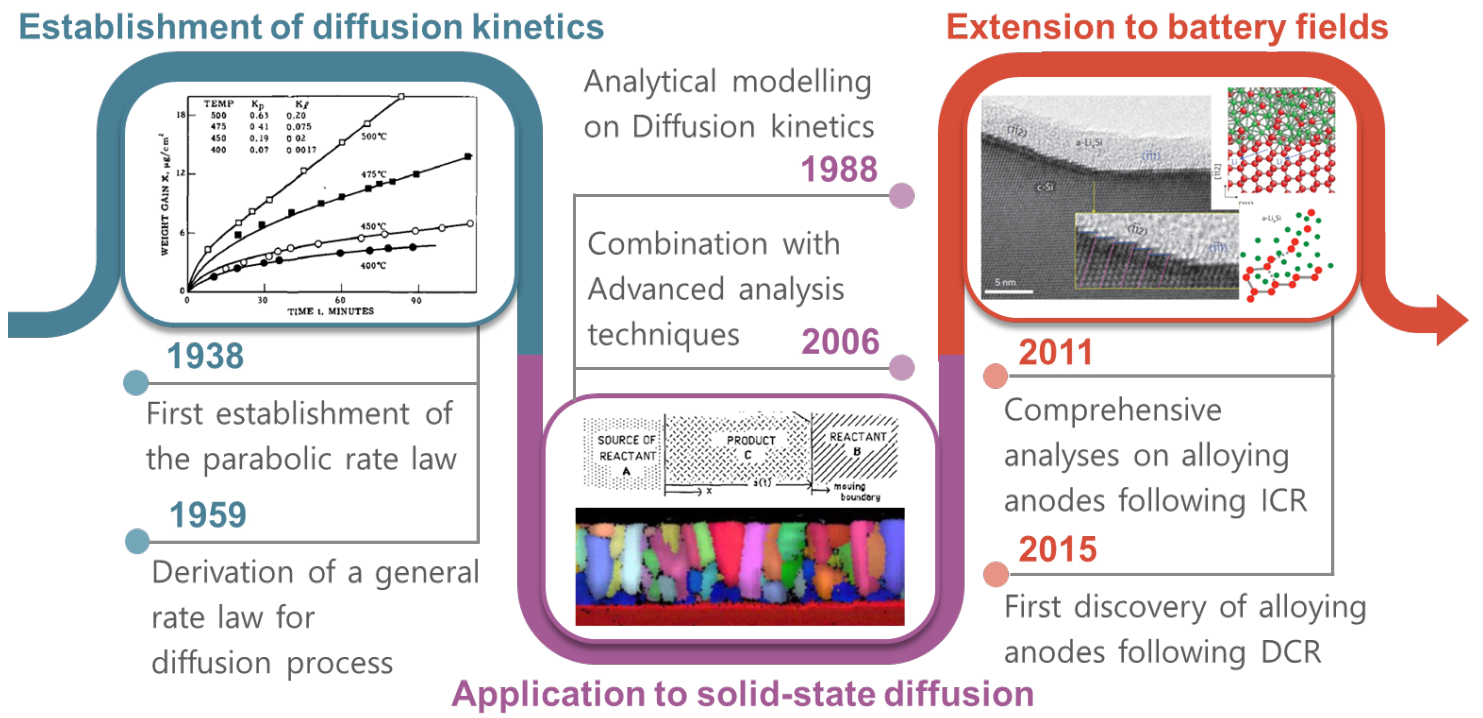

190

191 Figure 3. Schematic of the research history of diffusion kinetics, showing the establishment of the

192 theories on diffusion kinetics and its recent application to alloying anodes [34, 42, 47]. 


\section{Relationship between diffusion kinetics and anode performance}

Lack of capacity retention, slow charging rate, and short cycling life, which are directly related to the diffusion mechanisms operating in alloying anodes, are the major challenges that limit the commercialization of such anodes. Considerable effort has been exerted to address these issues in both LIBs ${ }^{[48]}$ and Na-ion batteries (NIBs) ${ }^{[49]}$. Of the various morphologies of anode materials, samples in the form of NWs are the most common shapes for analyzing the related diffusion kinetics because of two reasons. (1) One-dimensional NWs allow the direct tracking of the moving interface and associated analyses of the $L$ vs. $t$ relation. (2) Compared with film- or particle-type anodes, NW-shaped anodes accumulate comparatively low residual stresses during battery cycling. Anode samples with low residual stresses are particularly important for studying the diffusion kinetics intrinsic to anode materials because residual stresses can change the shape of the $L$ vs. $t$ curve and cause a system to seem governed by different diffusion kinetics. For example, in a Si particle, the shape of the $L$ vs. $t$ curve associated with lithiation is greatly affected by residual stresses whose magnitude is inversely proportional to the particle diameter; in principle, the lithiation of Si is governed by ICR and should exhibit the $L \propto t$ relation during lithiation (Section 2.2). However, the lithiation of Si particles appears to follow the parabolic rate law $\left(L^{2} \propto\right.$ t) because of the residual stresses accumulated at the $\mathrm{Si} / \mathrm{Li}_{x} \mathrm{Si}$ interface in particle-shaped $\mathrm{Si}$ anodes [50]. Therefore, cylindrical anode materials should be used to minimize the residual stresses developed at the interface and eliminate the associated artifacts. For this reason, this review is mainly conducted from the perspective of NW-shaped alloying anodes and summarizes how their diffusion behaviors and corresponding diffusion kinetics are related to such anodes' electrochemical properties, such as cycle life, rate performance, and specific capacity. 


\section{3.1. Diffusional anisotropy and cycle life of anodes}

The structure of anodes suffers from continual degradation during battery cycling, which

219 reduces the cycle life of the anodes. Of the various factors affecting the structural degradation of

220 anodes, orientation-dependent volume expansion is the most detrimental. For alloying anodes,

221 especially those following ICR diffusion, the difference in volume expansion can differ by up to

$222 \sim 400 \%$, depending on the crystallographic orientation. This variation causes the alloying anodes

223 to swell anisotropically. Large anisotropic swelling induces inhomogeneous stresses in alloying

224 anodes, which in turn cause mechanical degradation, as revealed by crack formation and anode

225 pulverization ${ }^{[19,51]}$. Because the anisotropic swelling behavior of anodes is closely related to the

226 diffusion kinetics of anode materials, we compare the degree of anisotropic swelling of anodes

227 governed by different diffusion kinetics and assess the relationship between the diffusion kinetics

228 and cyclability of alloying anodes.

229

Systems governed by ICR: Until the early 2000s, anisotropic solid-state diffusion was

231 known to occur only for materials with a non-cubic crystal symmetry. This is because, for systems

232 defined in continuum theory, the properties of crystals with a cubic symmetry are characterized by

233 a zero-rank tensor (i.e., scalar value) and thus are isotropic ${ }^{[52]}$. However, the model based on

234 continuum theory was found to no longer hold for the diffusion behaviors of alloying anodes. The

235 best example that violates the conventional rule is the anisotropic swelling of crystalline Si with a

236 diamond cubic symmetry ${ }^{[19,37]}$; observations of lithiated Si NWs revealed anomalous volume

237 changes wherein the shape of fully lithiated Si NWs differs depending on the crystallographic

238 orientations of the NWs (Figure 4a) ${ }^{[19,37]}$. Subsequent TEM analyses were performed on a Si/Li $\mathrm{i}_{x} \mathrm{Si}$

239 interface to understand the physics underlying these observed anisotropic swelling behaviors 
240 (Figure 4b). Observations of lithiated $\mathrm{Si} N W s$ revealed that the $\mathrm{Si} / \mathrm{Li}_{x} \mathrm{Si}$ interface is atomically

241 sharp and thin ${ }^{[18]}$ and propagates toward unreacted Si by defoliating close-packed Si (111) planes

$242{ }^{[42]}$. This defoliation, which is associated with the orientation-dependent, short-range diffusion near 243 the interface, causes the propagation speed of the interface to differ according to the 244 crystallographic orientation, resulting in anisotropic swelling.

245 The above discovery on the ICR diffusion of $\mathrm{Li} / \mathrm{Si}$ systems facilitated computational studies 246 on the reactions occurring at the $\mathrm{Si} / \mathrm{Li}_{x} \mathrm{Si}$ interface. On the basis of this observed defoliation 247 phenomenon, Liu et al. established an elasto-plastic deformation model and showed that the 248 anisotropic lithiation of $\mathrm{Si} N W s$ is attributed to the orientation of $\mathrm{Si} N W s$ at the $\mathrm{Si} / \mathrm{Li}_{x} \mathrm{Si}$ interface $249{ }^{[42]}$. Furthermore, the measurements of the propagation speed of the interface enable predicting the 250 morphological changes of Si NWs during lithiation. Using measured lithiation rates, an FEM study 251 calculated the $\mathrm{Li}$ diffusivity at the $\mathrm{Si} / \mathrm{Li}_{x} \mathrm{Si}$ interface, followed by a simulation of anisotropic 252 swelling and crack formation (Figure 4c) ${ }^{[53]}$. To further understand the reactions occurring at the 253 interface, atomic simulations based on molecular dynamics (MD) were performed to elucidate the 254 changes in atomic structures and stress states at the $\mathrm{Si} / \mathrm{Li}_{x} \mathrm{Si}$ interface during lithiation. This 255 approach allowed the validation of the previously reported defoliation behaviors of Si (111) planes 256 while assessing residual stresses and their effect on the anisotropic diffusion behavior (Figure 4d) $257{ }^{[54,55]}$. Recent works on the diffusion energy barrier at the $\mathrm{Si} / \mathrm{Li}_{x} \mathrm{Si}$ interface show that anisotropic 258 diffusion may arise from the orientation-dependent thermodynamic stability of the $\mathrm{Si} / \mathrm{Li}_{x} \mathrm{Si}$ 259 interface rather than from diffusion barriers for Li diffusion across the interface ${ }^{[56,57]}$. Despite this 260 unsettled debate, subsequent density functional theory (DFT) calculations further elaborated the 261 computational results by revealing the preferential pathways for Li diffusion and associated 262 diffusion barriers; Li ions tend to migrate along a direction parallel to the Li concentration gradient 
263 by taking regions or spaces with low valence electrons ${ }^{[58-60]}$. According to this simulation study, 264 the tortuosity of diffusion pathways is the structural origin of the anisotropic Li diffusion behavior $265[61]$.

266 Analyses of the anisotropic swelling behavior of Si NWs triggered extensive studies on the 267 anisotropic diffusion of other alloying anodes. In-situ TEM observations on the lithiation behaviors 268 of black phosphorus $(\mathrm{P})$ show that the transformation from orthorhombic $\mathrm{P}$ to amorphous $\mathrm{Li}_{x} \mathrm{P}$ is 269 accompanied by a large anisotropic volume expansion and associated crack formation (Figure 4e) $270{ }^{[62]}$. Similar results are observed when $\mathrm{Li}$ is substituted with $\mathrm{Na}$; during sodiation, crystalline $\mathrm{P}$ 271 expands preferentially along the [010] direction (Figure 4f) ${ }^{[63,64]}$. In addition to crystalline $\mathrm{P}, \mathrm{Sb}$ 272 also expands anisotropically upon sodiation ${ }^{[51]}$. All these systems follow the $L \propto t$ relation during 273 lithiation/sodiation. Thus, ICR diffusion is characterized by short-range diffusion and strongly 274 correlated with diffusional anisotropy. 
a

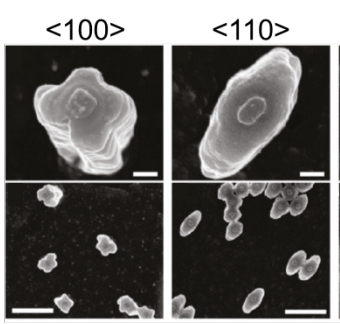

C $[11 \overline{1}]$

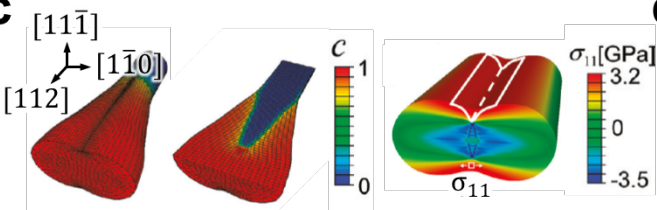

e
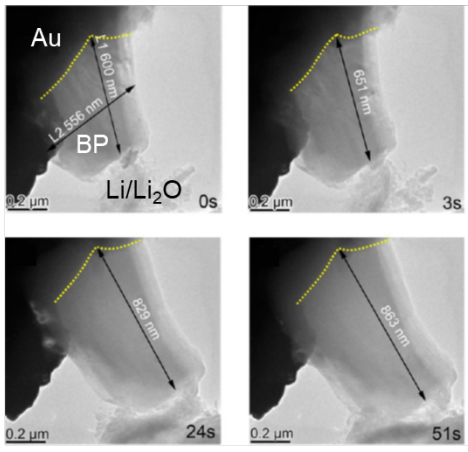

b

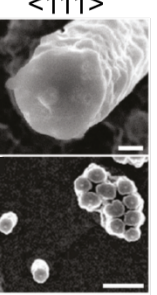

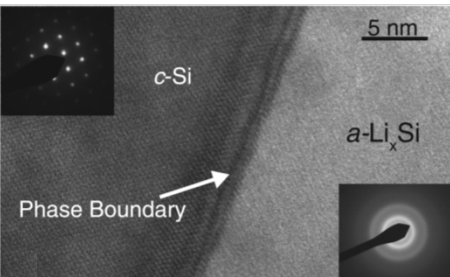

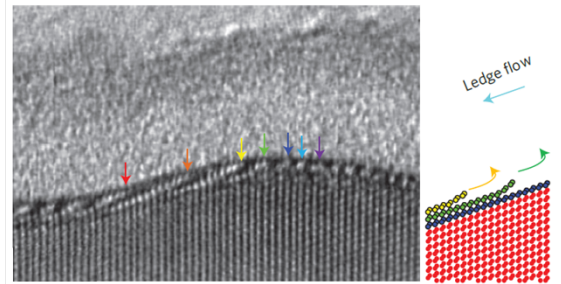

d

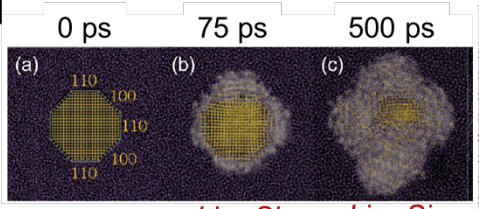

$L i_{0.89} \mathrm{Si}$

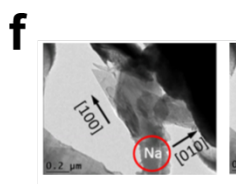

$\mathrm{Li}_{2.51} \mathrm{Si}$
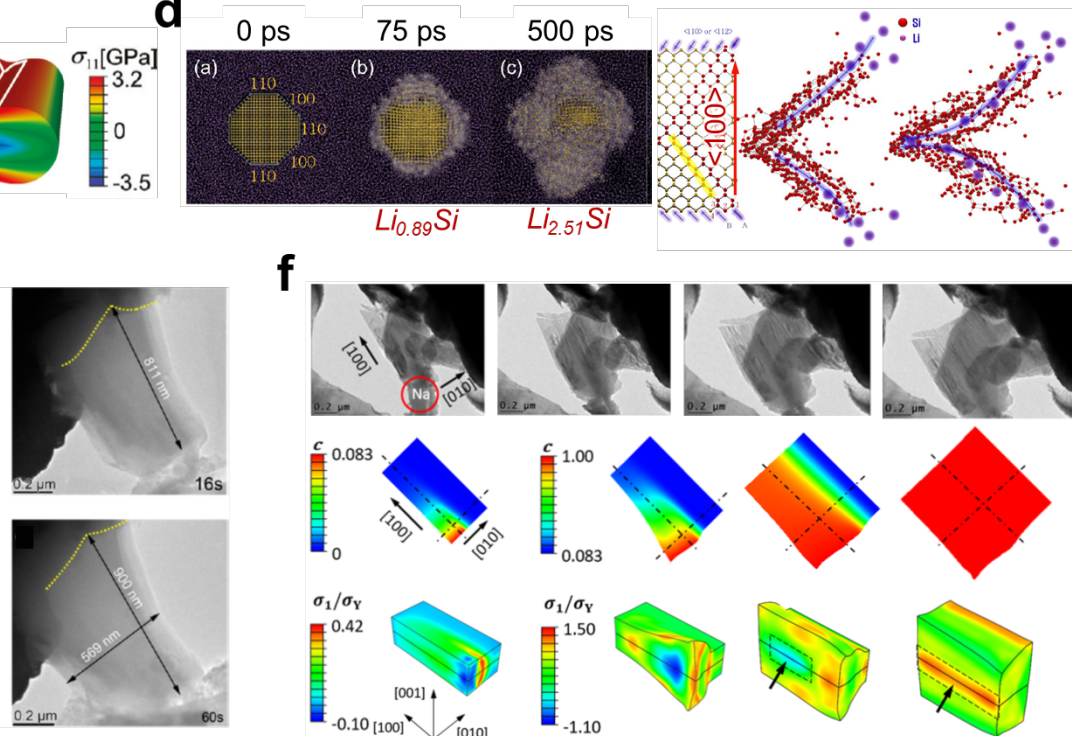
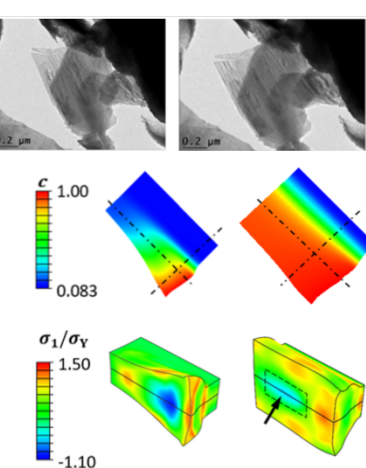

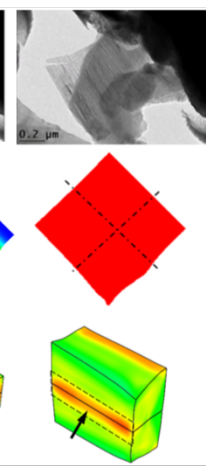

Figure 4. (a) SEM images of Si NWs subjected to lithiation, showing different morphological

278 changes depending on the initial crystallographic orientation of the Si NWs ${ }^{[37]}$. (b) High-resolution

279 TEM images of the atomically sharp interface between crystalline $\mathrm{Si}$ and lithiated $\mathrm{Li}_{x} \mathrm{Si}^{[18,42]}$,

280 showing the defoliation of close-packed Si (111) planes. (c) FEM simulation results on the

281 distribution of Li contents and residual stresses in the lithiated Si NWs ${ }^{[19]}$. (d) MD simulations

282 showing the morphological evolution of Si NWs associated with the lithiation ${ }^{[54]}$. (e) SEM images

283 of black P undergoing anisotropic swelling during lithiation ${ }^{[62]}$. (f) SEM images and FE analysis

284 on a partially sodiated $\mathrm{P}$ particle, showing the distribution of the $\mathrm{Na}$ contents and associated 285 residual stresses ${ }^{[64]}$. 
288

289

290

291

292

293

294

295

296

297

298

299

300

301

302

303

304

305

306

307

308

309

310

rate is limited by the diffusion of carrier ions in the trailing bulk region rather than by the diffusion at the interface. Consequently, the overall diffusion rate and associated swelling behaviors no longer depend on the crystallographic orientation of the anode material at the interface. Therefore, contrary to alloying anodes governed by ICR, those governed by DCR display an isotropic volume expansion during battery cycling. For example, the sodiation of 500-nm-diameter Sn NWs expands at equal rates along the radial direction regardless of the crystallographic orientations, resulting in isotropic swelling (Figure 5a) ${ }^{[65]}$. Measurements of the propagating speed of the interface reveal that the interface advances by following the $L^{2} \propto t$ relation, which confirms that the diffusion of $\mathrm{Na}$ into crystalline $\mathrm{Sn}$ is governed by DCR. The sodiation and lithiation behaviors of Ge NWs also follow the $L^{2} \propto t$ law ${ }^{[45,46]}$, thus causing Ge NWs to expand in a nearly isotropic manner. These results obtained from Ge NWs suggest that DCR diffusion is valid for such systems.

Notably, the crystal structures of anode materials following DCR diffusion have little in common: Sn (body-centered tetragonal) and Ge (diamond cubic). This suggests that the diffusion behaviors of anode materials following DCR are less likely to be affected by their crystal structure and orientation. Therefore, structural changes other than orientation effects are responsible for DCR diffusion. In early studies on the isotropic swelling of lithiated Ge NWs, this behavior was initially interpreted by relating the lithiation rate to the etching rate during wet-etching experiments [45]. Experiments showed that, compared with the etching rate of Si, that of Ge was approximately isotropic ${ }^{[66,67]}$. This indirectly indicated that the mobility of the lithiated Ge region is less sensitive to the orientation of the crystalline Ge. Although this explanation provided insights for understanding the isotropic diffusion behavior associated with DCR, it lacked a theoretical basis to support the DCR diffusion occurring during the two-phase reaction.

To further elucidate the structural origin of DCR diffusion, DFT calculations were performed 
311 on a $\mathrm{Na} / \mathrm{Sn}$ diffusion couple while observing the structural evolution of crystalline Sn adjacent to

312 the interface formed during sodiation. Calculations showed that, as $\mathrm{Na}$ ions begin to inflow into

313 the crystalline Sn, the crystal structure of the Sn near the interface becomes prone to disruption,

314 forming a thin layer of an amorphous phase (Figure 5c) ${ }^{[44,65,68]}$. The structure of thus-formed

315 amorphous layer near the interface is similar regardless of the initial orientation of crystalline $\mathrm{Sn}$;

316 this weakens or nullifies the directionality of $\mathrm{Na}$ diffusion and promotes isotropic diffusion. These

317 results indicate that isotropic diffusion can readily occur for alloying anodes with lower

318 interatomic bond energies. Therefore, such materials would require less energy to disrupt their

319 initial crystalline structures and thus facilitate amorphization behaviors. As explained in detail in

320 Section 3.2, the amorphization at the interface is an important structural origin that not only causes

321 isotropic diffusion but also accelerates the diffusion of carrier ions. This renders the overall

322 diffusion rate to be controlled by the trailing bulk region, i.e., DCR diffusion.

323 In addition to low bond energies, the diameter of NWs can promote the formation of 324 amorphous phases in front of the advancing interface and the associated isotropic diffusion. For 325 instance, a previous study on the oxidation behavior of Si NWs reported that the pristine Si core 326 does not act as a rigid mechanical constraint during oxidation when the diameter of Si NWs is 327 smaller than $5 \mathrm{~nm}{ }^{[69]}$. As a result, for small Si NWs, the crystalline structures of the Si core can 328 be readily disrupted to reduce residual stresses during oxidation (Figure 5d). This behavior of 329 amorphization weakens the orientation-dependent diffusion in Si NWs, thus enabling the oxidation 330 to proceed in an isotropic manner. Amorphization behaviors similar to Si are observed from 331 various metal NWs, whereas their threshold diameter for promoting the amorphization behavior 332 differs depending on the materials ${ }^{[70]}: 300 \mathrm{~nm}$ for $\mathrm{Si}^{[37,71]}, 1200 \mathrm{~nm}$ for ${ }^{[72}{ }^{[72]}$, and $\sim 60 \mathrm{~nm}$ for $333 \mathrm{CuO}{ }^{[73]}$. 
The above experimental and simulation results suggest that, in principle, DCR diffusion and

335 the associated isotropic swelling are feasible for group IVA and VA elements with small diameters

336 and interatomic bond energies. Considering that isotropic swelling can prevent the accumulation

337 of high residual stresses and crack formation ${ }^{[70]}$, the cyclability of alloying anodes could be

338 improved by decreasing their size to below the threshold diameter. However, the synthesis of

339 nanoscale anode materials is challenging and costly because of their susceptibility to external

340 contaminants, such as oxygen and moisture ${ }^{[74]}$. Therefore, given the cost and complexity of anode

341 fabrication, alloying anodes with large threshold diameters are desirable for producing long-lasting 342 alloying anodes.

a
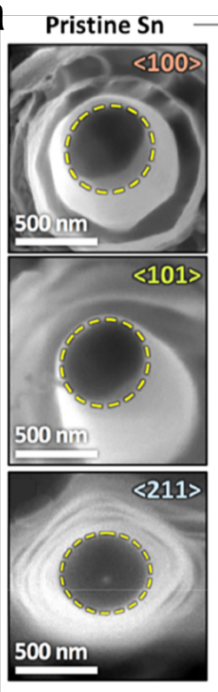

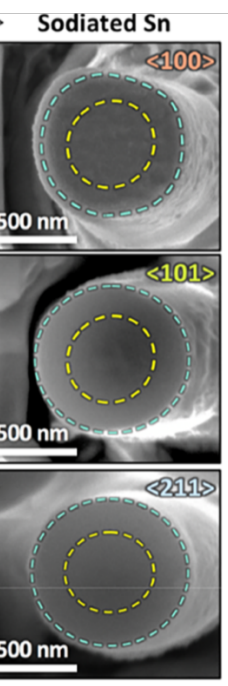

b
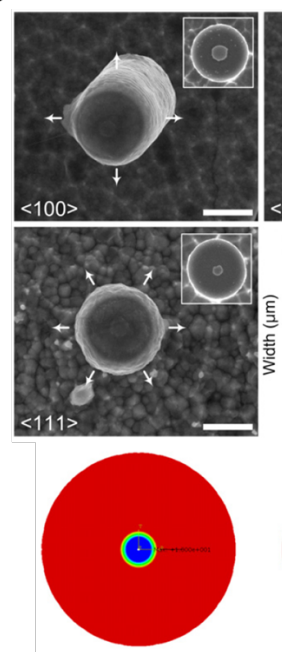

C

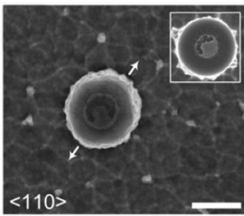

C

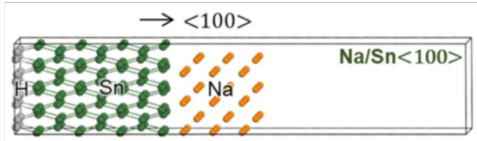

$\mathrm{Na} / \mathrm{Sn}<100>$

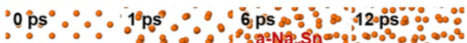
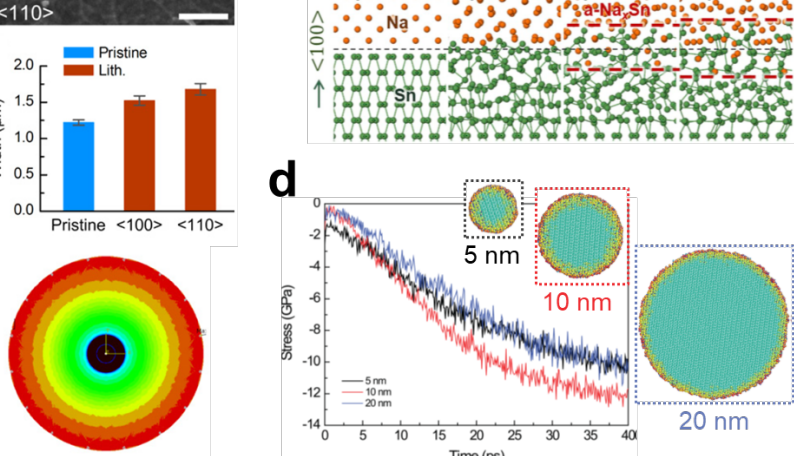

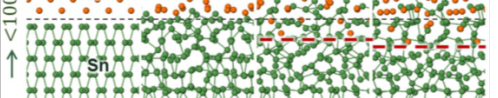

d

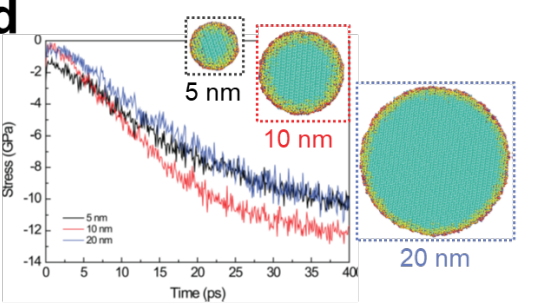

345 Figure 5. SEM images of the (a) sodiated Sn ${ }^{[65]}$ and (b) lithiated Ge NWs ${ }^{[72]}$, showing that these

346 materials expand isotropically regardless of their crystallographic orientation during

347 sodiation/lithiation. (c) DFT calculations performed on the $\mathrm{Na} / \mathrm{Sn}$ diffusion couple, displaying that

348 sodiation of Sn proceeds by the formation of a thin amorphous Sn layer in front of the advancing

349 interface ${ }^{[44]}$. (d) Distribution of residual stresses of partially oxidized Si NWs, showing the release

350 of residual stresses in Si NWs with diameters smaller than $5 \mathrm{~nm}{ }^{[69]}$. 


\subsection{Diffusion rate of carrier ions and rate performance}

Compared with insertion electrodes, alloying anodes display slow diffusion rates of carrier

354 ions; thus, batteries exhibit poor rate performance. Reduced diffusion rates within alloying anodes

355 arise from the characteristic diffusion mechanism of carrier ions operative in these anode materials.

356 The width of diffusion channels, where carrier ions can diffuse, is not large in alloying anodes,

357 unlike that in insertion electrodes. Hence, the carrier ions within alloying anodes diffuse by

358 breaking the atomic bonds of the host anode material, leading to the disruption of the crystal

359 structures. This process requires additional energy and thus significantly lowers the diffusion rate

360 of carrier ions. Therefore, the disruption process of the crystal structure of anode materials needs

361 to be analyzed to understand the diffusion rate and associated rate performance of alloying anodes.

362 In this section, the structural changes occurring in alloying anodes are compared for systems

363 following ICR and DCR diffusion, and the relationship between the diffusion mechanism and 364 diffusion rate of carrier ions is discussed.

Systems governed by ICR: Previous studies on diffusion in alloying anodes found that

367 systems such as $\mathrm{Li} / \mathrm{P}^{\left[{ }^{62}\right]}, \mathrm{Na} / \mathrm{Sb}{ }^{[44]}$, and $\mathrm{Li} / \mathrm{Si}{ }^{[19]}$ are controlled by ICR (Figure 6a). For these

368 systems, the diffusion rate is governed by the diffusion of carrier ions at the propagating interface,

369 resulting in low diffusion rates of carrier ions. In general, this characteristic diffusion is related to

370 the two structural features of the propagating interface, i.e., 1) the atomically sharp/narrow

371 interface ${ }^{[75,76]}$ and 2) the curvature of the interface ${ }^{[40,50,75]}$.

372 The presence of an atomically sharp interface in ICR-governed systems implies that the 373 composition of carrier ions changes rapidly across the interface. This change in the composition 
374 induces a large misfit strain and develops high compressive residual stresses to the unreacted anode

375 material near the interface ${ }^{[18]}$. Compressive residual stresses $(\sigma)$ in turn increase the activation

376 energy (or diffusion barrier) for diffusion by a certain amount $(\sigma \Delta V)$, which reduces the diffusivity

377 and hinders the additional inflow of carrier ions into the anode. This is often revealed in

378 experiments as a phenomenon called the "self-limiting diffusion" behavior ${ }^{[77-79]}$ and is explained

379 in detail in Section 3.3. One way to mitigate the formation of a sharp interface is to utilize anodes

380 with amorphous phases. For example, compared with crystalline Si with a sharp interface, an

381 amorphous structure with a broad interface can transport Li ions into the Si anode at faster rates.

382 This is because amorphous $\mathrm{Si}$ is characterized by loosely packed atomic structures (of Voronoi

383 polyhedral ${ }^{[80]}$ ) and thus can readily transport Li ions through an amorphic interface (Figure 6b).

384 Another important parameter that limits the diffusion rates of alloying anodes is the curvature

385 of the propagating interface. Compared with a flat/straight interface, a curved interface develops

386 additional compressive stresses in the direction tangent to the interface during the diffusion of

387 carrier ions. The increment in stress is linearly related to the curvature (or inversely proportional

388 to the radius) of the interface, thereby reducing the diffusion rates of carrier ions at the interface.

389 A subsequent model study quantified this decrease in the diffusion rate by relating the residual

390 stress at the interface with the increase in Gibbs free energy ${ }^{[40]}$. This model was later used to

391 analyze the residual stresses generated within Si particles during lithiation and the corresponding

392 changes in the lithiation rate (Figure $6 c)^{[50]}$, and the results were subsequently validated through

393 experiments ${ }^{[81]}$. 

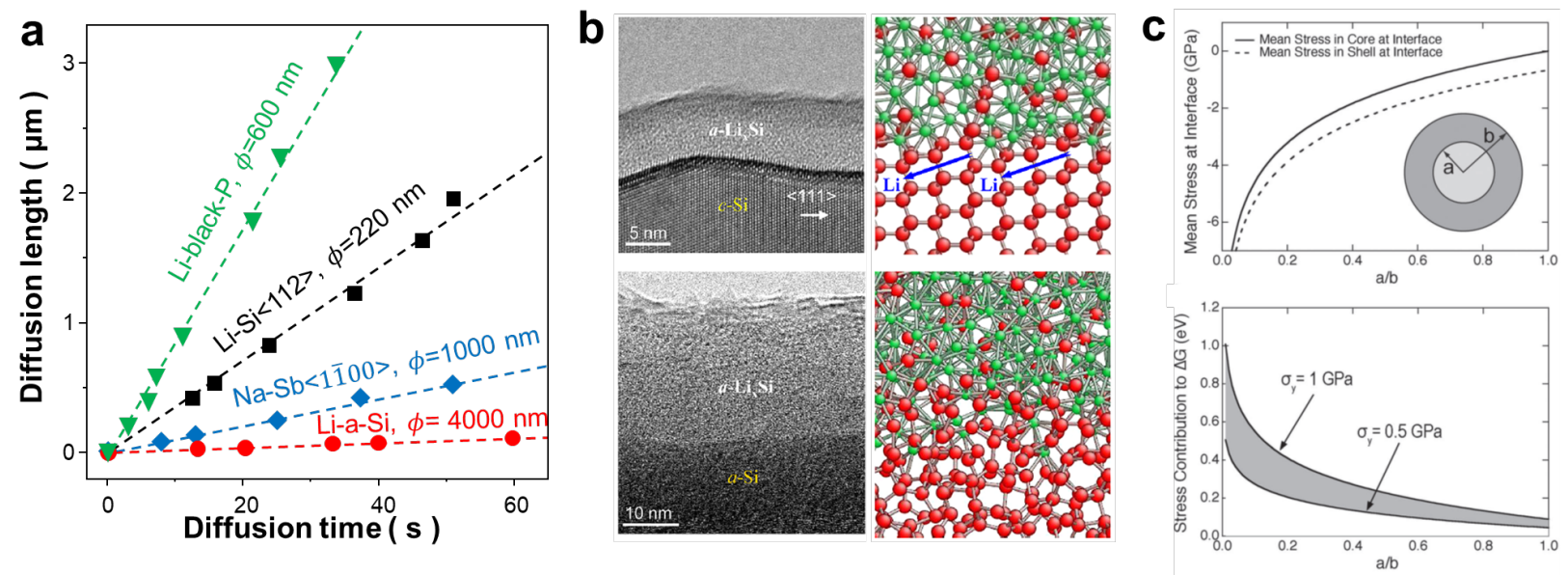

Figure 6. (a) The $L$ vs. $t$ graphs constructed for various alloying anodes following ICR $[19,44,62,76]$.

(b) TEM images and MD simulation results, showing different interfacial structures observed from crystalline and amorphous Si subjected to lithiation ${ }^{[76]}$. (c) Changes in the residual stress and the corresponding changes in Gibbs free energy calculated as a function of lithiated layer thickness in the Si particle ${ }^{[50]}$.

Systems governed by DCR: With the progress of researches on alloying anodes, alloying anodes that cannot be explained by the ICR diffusion mechanism were discovered for the Na-Sn ${ }^{[82]}$ and Li-Ge ${ }^{[45]}$ systems. These systems commonly follow the $L^{2} \propto t$ law in solid-state diffusion (Figure 7a), whereas the propagation rates of the interface were faster by $2-3$ orders of magnitude than those of alloying anodes following ICR diffusion (Figures 6a and 7a). The $L^{2} \propto t$ relationship and fast diffusion rates of carrier ions suggest that the diffusion of carrier ions in these systems is not restricted by the propagating interface, a characteristic of the DCR diffusion mechanism. observed that DCR diffusion arises from the formation of an amorphous phase and dislocation

411 cloud near the propagating interface during Li diffusion (Figure 7b). Such structural changes, 
412 especially dislocation generation, are induced because of the initial preference of Li diffusion

413 along the [001] direction of $\mathrm{SnO}_{2}$, which causes a $\sim 10 \%$ lattice expansion in the (100) planes ${ }^{[83]}$.

414 These dislocations and amorphous phases act as fast diffusion pathways of Li ions ${ }^{[84]}$ and thus can

415 carry Li ions to regions away from the interface, thereby enabling long-range diffusion, which is

416 a characteristic of DCR. The formation of dislocation clouds and the long-range diffusion behavior

417 of carrier ions are also observed from other oxide anode materials, such as $\mathrm{ZnO}{ }^{[85]}$ and $\mathrm{RuO}_{2}{ }^{[86]}$.

418 Theories explaining ICR diffusion can be extended to explain the diffusion behaviors of

419 alloying anodes following DCR diffusion. For a Na/Sn system, the propagation length $(L)$ of the

420 interface measured from Sn NWs follows the $L^{2} \propto t$ law, suggesting that the sodiation of Sn is

421 governed by DCR (Figure 7a). Compared with systems following ICR, systems following DCR

422 commonly exhibit a high diffusivity of carrier ions and thus improved rate performance. The

423 enhanced diffusivity measured from DCR-governed anode materials arises from two different

424 structural features: the formation of a thin amorphous layer and dislocations in front of the

425 interface. Byeon et al. performed ab initio MD simulations on a $\mathrm{Na} / \mathrm{Sn}$ system to quantitatively

426 explain how the amorphization can promote the diffusivity of carrier ions in the system following

427 DCR ${ }^{[65,68]}$. Calculations showed that the amorphization increases the interatomic bond distance

428 of the adjacent $\mathrm{Sn}-\mathrm{Sn}$ pairs near the interface (Figure 7c). Therefore, the amorphization at the

429 interface causes the phase to reveal atomically open spaces. These spaces provide a preferential

430 passage for $\mathrm{Na}$ diffusion and thus facilitate the fast transport of $\mathrm{Na}$ ions at the interfacial region.

431 When viewed at the macroscopic scale, the amorphization at the interface alleviates the rate-

432 limiting behavior of the propagating interface and causes the overall kinetics of the system to

433 follow DCR diffusion. Additional advanced analyses performed at the propagating interface reveal

434 that dislocations formed within crystalline Sn act as preferential pathways for Na diffusion (Figure 
$4357 \mathrm{c})^{[65]}$.

436 Both the amorphization and dislocation generation mitigate the residual stresses developed

437 on the interface. Consequently, anodes following DCR diffusion exhibit an ultrafast charging rate

438 and alleviated self-limiting diffusion. Furthermore, dislocations generated near the propagating

439 interface can act as a preferential pathway for diffusion because the activation energy for diffusion

440 is lowered upon diffusion through dislocation cores. This accelerates the diffusion of carrier ions

441 and facilitates the long-range diffusion of carrier ions at accelerated rates, which is typically known

442 as "dislocation-pipe diffusion" [84, 87-89]. The transport rate of atoms during pipe diffusion is many

443 orders of magnitude faster than that during bulk diffusion in a crystal ${ }^{[84,90]}$. Therefore, in addition

444 to the formation of a thin amorphous layer in front of the interface, dislocation-pipe diffusion in

445 alloying anodes is an important mechanistic origin supporting the high rate performance of anode

446 materials following DCR diffusion. 

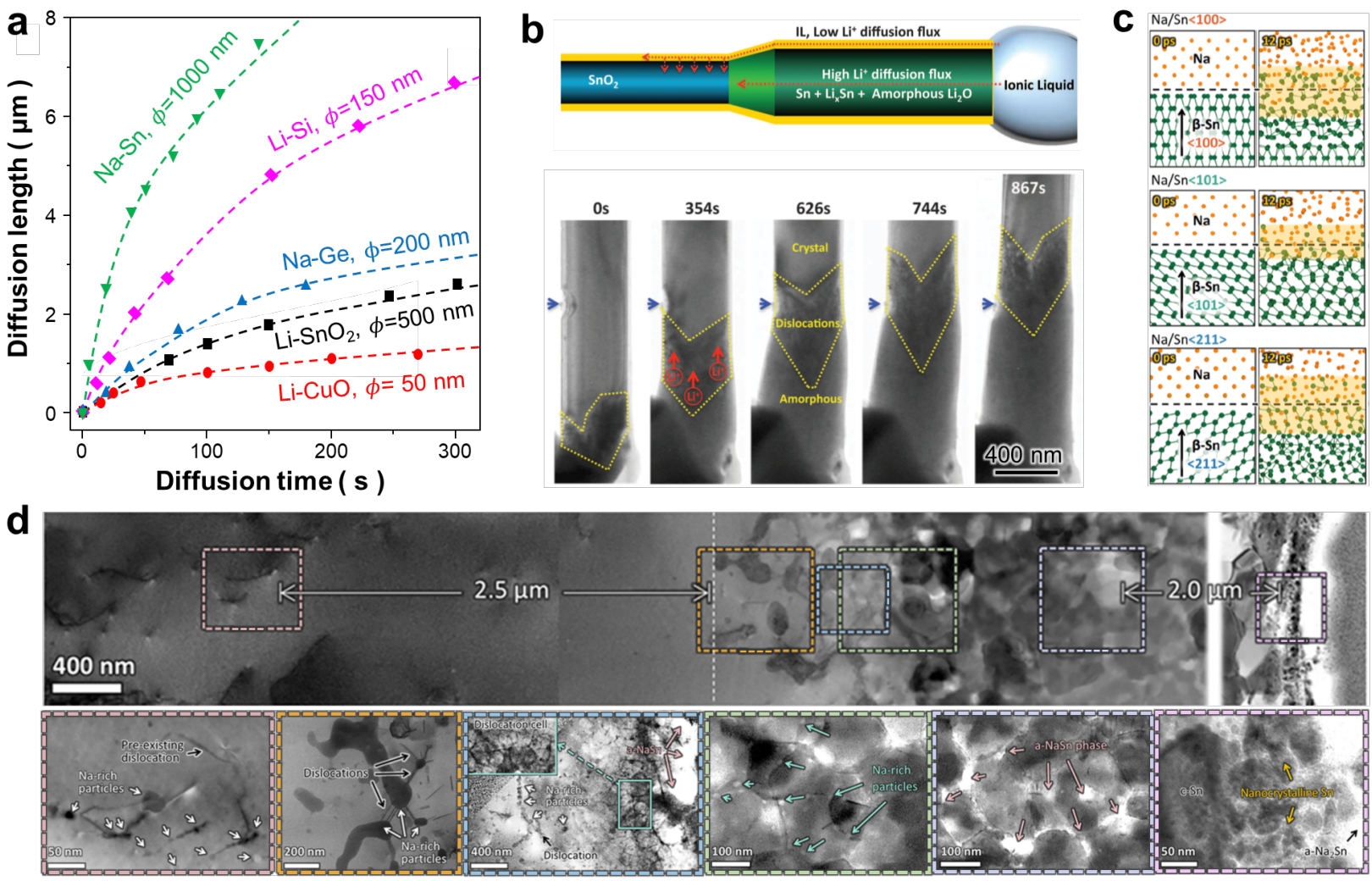

Figure 7. (a) $L$ vs. $t$ graphs constructed for various alloying anodes following DCR [19, 36, 46, 73, 82].

450 (b) Formation of dislocations in front of the propagating interface captured during lithiation of

$451 \mathrm{SnO}_{2} \mathrm{NWs}$, explaining the long-range diffusion of Li ions ${ }^{[36]}$. (c) DFT calculations showing the

452 amorphization behavior of crystalline Sn near the interface [65]. (d) TEM images showing the

453 formation of dislocations in crystalline Sn at regions away from the interface during sodiation ${ }^{[65] .}$ 


\subsection{Carrier ion penetration depth and specific capacity}

Despite its large theoretical capacity, the alloying anode can only utilize limited capacity during battery cycling (Figure 1). This low practical capacity is mainly attributed to the short penetration depth of carrier ions into the anode material because the diffusion of carrier ions is often arrested or self-limited inside the anode material, which hinders its full utilization. This behavior, referred to as self-limiting diffusion (SLD), reveals itself as the limited practical capacity of alloying anodes ${ }^{[77,91]}$. Numerous efforts to understand the SLD behavior showed that it stems from a large volumetric expansion and the development of residual stresses associated with the diffusion of carrier ions ${ }^{[91,92]}$. Therefore, the development of anodes with large energy capacity relies on the ability to control the residual stresses developed in the alloying anodes.

Previous research on the diffusion behavior of alloying anodes reported that the residual stresses developed in the anodes are closely related to the diffusion kinetics of carrier ions (Sections 3.1 and 3.2). In this regard, diffusion kinetics can be a key concept for interpreting the SLD behavior encountered in alloying anodes. In this section, we compare the SLD behavior of alloying anodes governed by ICR and DCR diffusion kinetics and assess the relationship between the diffusion kinetics and the practical capacity of alloying anodes.

Systems governed by ICR: $\mathrm{Si}$, the most well-known alloying anode following ICR, is known to exhibit SLD behavior with various solute atoms, including $\mathrm{O}^{[69]}, \mathrm{Li}^{[77]}$, and $\mathrm{B}{ }^{[93]}$. For the battery systems, SLD behavior occurring in the Li/Si couple began to gain attention in TEM studies performed on the lithiation of Si NWs ${ }^{[94]}$. Experiments showed that lithiated Si NWs form a core-shell structure consisting of unreacted $\mathrm{Si}$ at the interior and amorphous $\mathrm{Li}_{x} \mathrm{Si}$ at the outer layer. In the early 2010s, extensive TEM research was conducted to observe the SLD behavior of 
478 Si NWs and Si NPs during lithiation. Liu et al. ${ }^{[77]}$ observed the lithiation behavior of Si by directly 479 attaching an $\mathrm{Li}_{2} \mathrm{O} / \mathrm{Li}$ electrode to a 129 -nm-diameter $\mathrm{Si} \mathrm{NW}$ and traced the movement of the 480 reaction front (Figure $8 \mathrm{a}$ ). The reaction front initially moved rapidly toward the interior of the $\mathrm{Si}$ 481 NW, after which it gradually slowed down, before stopping completely after 1.5 hours of lithiation. 482 This caused the lithiation of the Si NW to be arrested after penetrating only $55 \%$ of the entire 483 volume of the Si NW. Model studies showed that the observed SLD phenomenon arises from the 484 development of large compressive residual stresses at the sharp interface. Si NPs also suffer from 485 the SLD behavior similar to that observed in Si NWs ${ }^{[50]}$; in-situ TEM studies showed that for all 486 Si NPs with diameters of 90-200 nm, lithiation is impeded when the lithiated volume of Si NPs 487 reaches approximately $50 \%$ (Figure $8 \mathrm{~b}$ ).

488 The SLD behavior of Si crystals can be explained by the increase in the Gibbs free energy $489(\Delta G)$ estimated for the consumption of one $\mathrm{Li}$ ion to form $1 / x$ units of $\mathrm{Li}_{x} \mathrm{Si}$ :

$$
\Delta G=\Delta G_{r}-e \phi+\frac{1}{x}\left[\bar{\sigma}_{S i} V_{S i}-\bar{\sigma}_{L i_{x} S i} V_{L i_{x} S i}\right]
$$

491 where $\Delta G_{r}$ is the free energy change in the absence of mechanical stress $(\sigma)$ and applied voltage $492(\phi)$. The second term $(-e \phi)$ is the free energy decrease in the presence of the voltage applied to 493 the electrochemical cell, whereas the third term in brackets is the increase in the free energy owing 494 to the presence of mechanical stress in the structure, where $\bar{\sigma}_{S i}$ and $\bar{\sigma}_{L i_{x} S i}$ are the mean stress in 495 the $\mathrm{Si}$ core at the interface and in the lithiated silicon, respectively, whereas $V_{S i}$ and $V_{L i_{x} S i}$ 496 correspond to the volume of $\mathrm{Si}$ and $\mathrm{Li}_{x} \mathrm{Si}$, respectively. From an analysis of Eq. (1), the 497 compressive stresses increase with increasing thickness of the lithiated layer, which suppresses 498 further transportation of Li into Si NPs, and causes the SLD behavior. The SLD behavior was also 499 observed in micrometer-scale Si structures ${ }^{\left[{ }^{92]}\right.}$ and other alloying anodes governed by ICR [44]. 500 As discussed in the earlier Sections 3.1 and 3.2 and in detail in the following, high compressive 
501 residual stresses are observed to develop in regions near the interface of the systems following 502 ICR diffusion, suggesting that the SLD behavior can be attributed to this type of diffusion.

503 Although the above theories assisted in understanding of the relationship between the SLD 504 behavior and ICR diffusion, these models cannot fully reproduce the SLD behavior of certain 505 systems. For instance, the theories claimed that the SLD behavior of the Li/Si system occurs 506 regardless of the initial size of Si NWs or NPs ${ }^{[50]}$. However, experimental studies on the lithiation 507 of Si reported that, contrary to the model predictions, the penetration depth of carrier ions differs 508 depending on the initial diameter of Si NPs. The full penetration of Li ions was observed for Si 509 NPs with diameters smaller than $80 \mathrm{~nm}$ (Figure 8c) ${ }^{[71]}$, whereas only partial lithiation for $140-\mathrm{nm}$ 510 diameter Si NWs ${ }^{[43]}$. Subsequent studies interpreted this size-dependent SLD to arise, because the 511 amount of stress-induced energy $(\sigma V)$ accumulated near the interface was relatively small for $\mathrm{Si}$ 512 NWs with small diameters ${ }^{[71]}$. In addition to the above examples, the full lithiation of Si was also 513 observed in Si NPs with an amorphous structure (Figure 8d). The amorphous Si phase can readily 514 relieve the compressive residual stresses developed at the interface via structural relaxation, which 515 allows the lithiation to proceeds toward the Si core. The above examples demonstrating the full 516 lithiation of $\mathrm{Si}$ indicate that the numerical models based on the continuum theory alone are 517 insufficient to fully interpret the SLD behaviors. This is because, when viewed at the atomic scale, 518 the SLD behavior is closely related to the structural changes, such as amorphization ${ }^{[44,65]}$ and 519 dislocation generation ${ }^{[36,65]}$, which can partially relieve residual stresses. This will be explained 520 in detail in the following by relating the structural changes to the DCR diffusion. 

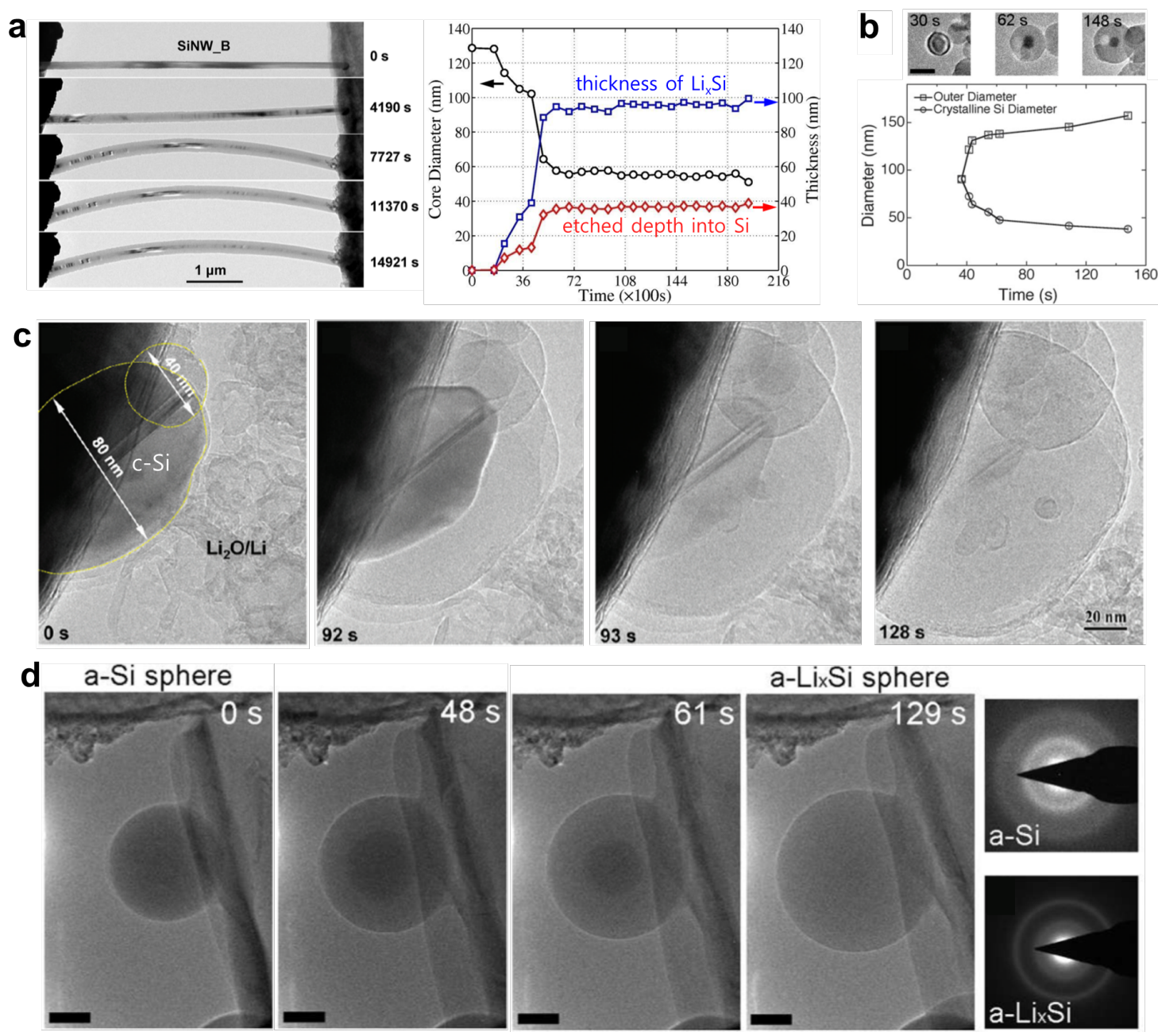

523 Figure 8. Self-limiting lithiation behavior observed in: (a) 129-nm-diameter Si NW [77] and (b)

52490 -nm-diameter Si NPs ${ }^{[50]}$. TEM images showing the full lithiation of Si NPs captured during

525 lithiation of: (c) crystalline Si NPs ${ }^{\text {[71] }}$, and (d) amorphous Si NPs ${ }^{[75]}$.

Systems governed by DCR: Of various anodes following DCR, Ge is the first material for

528 which the penetration behavior of carrier ions was observed. Lithiation experiments performed on

529 Ge showed that, while having the same crystal structure as Si, the SLD behavior is significantly

530 mitigated for Ge. When a 165-nm-diameter Ge NW was in contact with $\mathrm{Li}$, Li ions penetrated into

531 the core of the Ge NW, transforming Ge into $\mathrm{Li}_{15} \mathrm{Ge}_{4}$ (Figure 9a) ${ }^{[95]}$. In another experiment, the 
532 full lithiation in Ge NPs with various diameters of 160-620 nm was observed (Figure 9b) ${ }^{[96]}$. The

533 lithiation behavior of Ge crystals largely differ from those observed in Si crystals that display SLD

534 even for 130-nm-diameter Si NWs (Figure 8a). This suggests that the threshold diameter for SLD

535 is larger for Ge than for $\mathrm{Si}$, indicating that the anode materials following DCR diffusion can fully

536 utilize their theoretical capacity even for a relatively large size. Furthermore, close examination of

537 lithiated Ge revealed additional features, e.g., compared to the sharp and straight interface and 538 anisotropic expansion observed in the $\mathrm{Li} / \mathrm{Si}$ system, the $\mathrm{Li} / \mathrm{Ge}$ system displays an obscure and 539 tortuous interface and expands isotropically. This indicates that the atomic scale interactions 540 occurring at the $\mathrm{Ge} / \mathrm{Li}_{x} \mathrm{Ge}$ interface differ from those at the $\mathrm{Si} / \mathrm{Li}_{x} \mathrm{Si}$ interface.

To better understand the physics behind the SLD behavior, Park et al. ${ }^{[44]}$ performed a 542 comparative study on the $\mathrm{Na} / \mathrm{Sb}$ and $\mathrm{Na} / \mathrm{Sn}$ systems governed by ICR and DCR, respectively. They

543 observed the structural evolution near the interface of the two systems and established a 544 relationship between the diffusion kinetics and SLD behavior. Compared to the $\mathrm{Na} / \mathrm{Sb}$ system 545 (ICR), the $\mathrm{Na} / \mathrm{Sn}$ system (DCR) exhibits a much deeper penetration of $\mathrm{Na}$ into Sn, while forming 546 a tortuous and obscure $\mathrm{Sn} / \mathrm{Na}_{x} \mathrm{Sn}$ interface (Figure 9c). Subsequent DFT calculations showed that 547 in the $\mathrm{Na} / \mathrm{Sb}$ system, $\mathrm{Na}$ ions diffuse toward crystalline $\mathrm{Sb}$, while the initial crystal structure of $\mathrm{Sb}$ 548 is retained. This not only requires high energy for diffusion, but also leads to the development of 549 residual stresses near the interface, which arrests $\mathrm{Na}$ diffusion into Sb. Conversely, Na diffusion 550 in the $\mathrm{Na} / \mathrm{Sn}$ system proceeds by forming a thin amorphous layer in front of the advancing interface $551{ }^{[65]}$. The amorphization alleviates the residual stress development near the interface, which, in turn, 552 permits the additional penetration of $\mathrm{Na}$ ions into the $\mathrm{Sn}$ interior.

553 Another important structural evolution affecting the SLD behavior is the generation of 554 dislocations preceding an advancing interface. TEM observations near the interface of the $\mathrm{Na} / \mathrm{Sn}$ 
555 system showed that residual stresses developed in regions near the propagating interface can 556 generate dislocations near the interface ${ }^{[65]}$. Thus-formed dislocations in a Sn crystal not only 557 relieve the stress-induced energy, but also promote the transport of $\mathrm{Na}$ ions through dislocation 558 cores, by attracting $\mathrm{Na}$ ions into their open spaces. Continual supply of $\mathrm{Na}$ atoms via this 559 dislocation-pipe diffusion causes Na-rich particles to grow, which results in a localized plastic 560 yielding of the adjacent crystalline $\mathrm{Sn}$ grains by generating a dislocation burst. The repetition of 561 the pipe diffusion and generation of additional dislocations increase significantly the penetration 562 depth of $\mathrm{Na}$ ions, which mitigates the SLD behavior and improves the practical capacity of Sn 563 anodes.

564 The relationship between the SLD behavior and diffusion kinetics can further be explained 565 by relating the various experimental observations to the measured residual stresses near the 566 interfaces ${ }^{[97]}$. For the Li/Si system governed by ICR diffusion, lithiated Si develops a large misfit 567 strains and the associated compressive residual stresses near the $\mathrm{Si} / \mathrm{Li}_{x} \mathrm{Si}$ interface (Figure 9d). The 568 compressive stresses increase the stress-induced energy term $(\sigma V)$ in Eq. (1), which in turn 569 increases the overall driving force of Li diffusion and causes the SLD behavior. Conversely, for 570 systems governed by DCR, the diffusion of carrier ions is often accompanied by the disruption of 571 crystalline structures and generation of dislocations, which can reduce the stress-induced energy 572 term and thus increase the driving force of diffusion. This was confirmed by stress measurements 573 at the atomic scale performed on a partially sodiated Sn anode, where the residual stresses develop 574 near the $\mathrm{Sn} / \mathrm{Na}_{x} \mathrm{Sn}$ interface were much smaller than those measured at the $\mathrm{Si} / \mathrm{Li}_{x} \mathrm{Si}$ interface 575 (Figure 9e). Such low residual stresses cause Na ions to diffuse much deeper into Sn anode at fast 576 rates, which allows Sn anodes to fully utilize their theoretical capacity. 
a

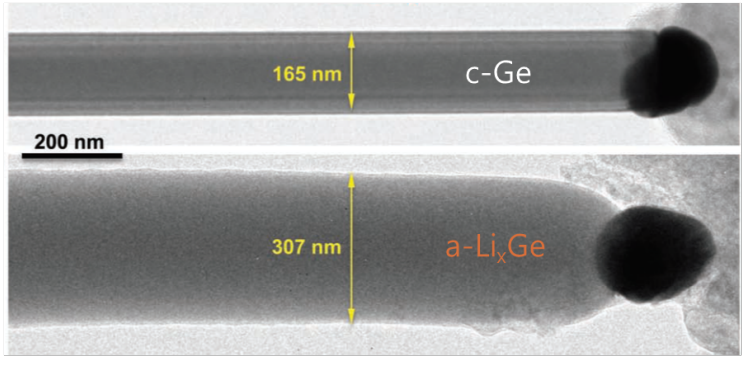

C
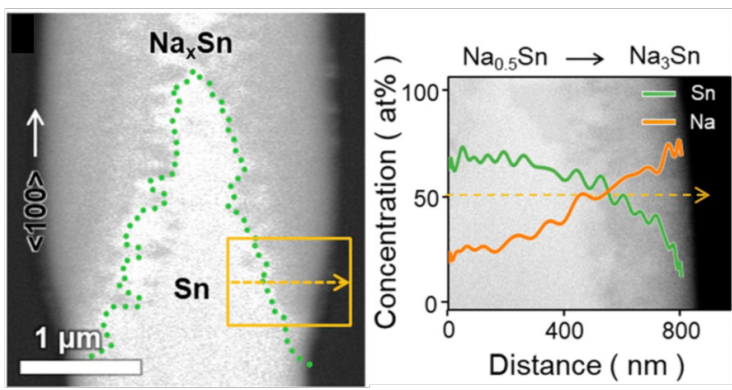
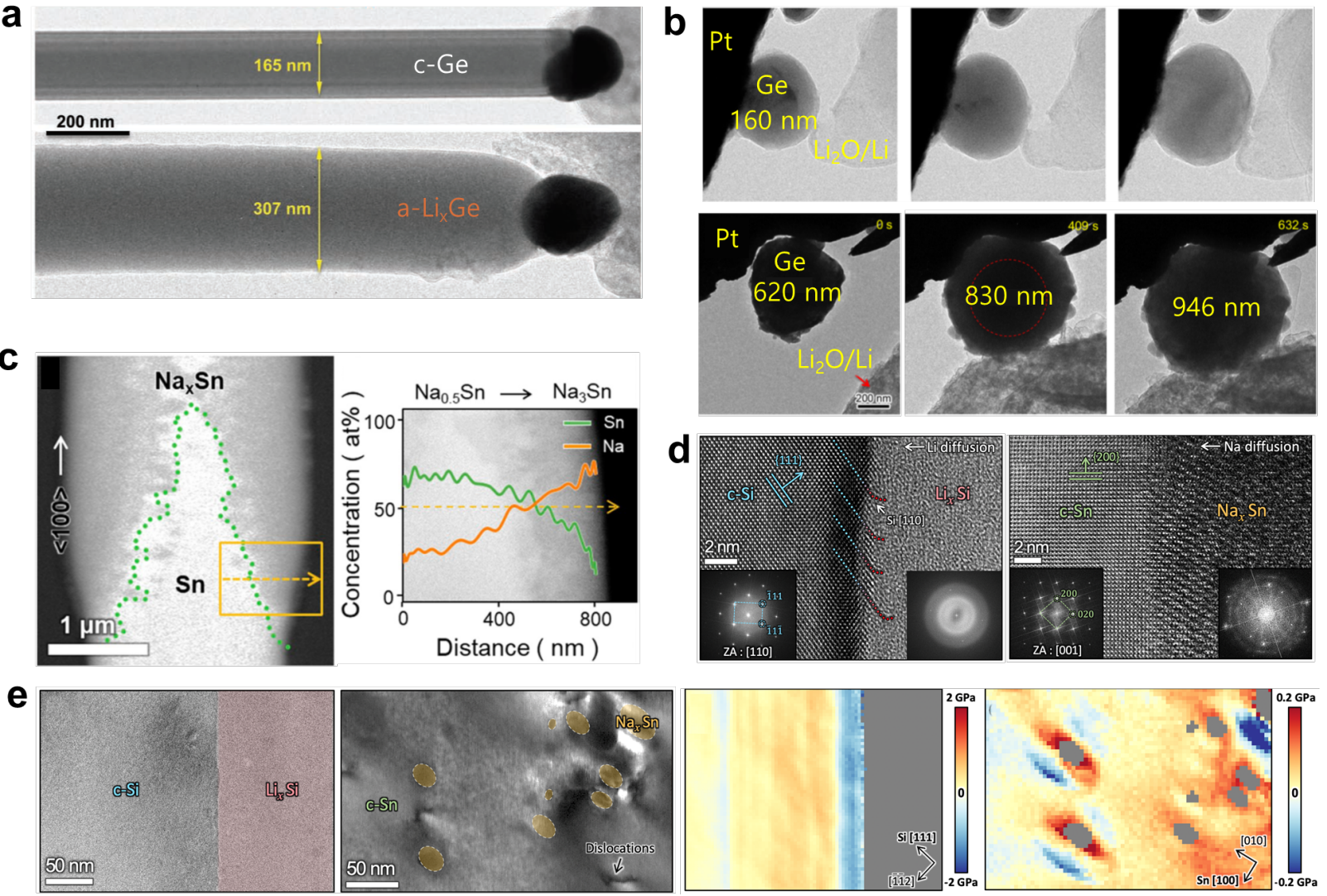

Figure 9. TEM images captured during in-situ lithiation experiments performed on: (a) a 165-nmimage and energy-dispersive X-ray spectroscopy line profiles recorded from longitudinal crosssection near propagating phase boundary of sodiated Sn pillar ${ }^{[44]}$. (d) High-resolution TEM images of interfacial structure recorded at $\mathrm{Si} / \mathrm{Li} x_{x} \mathrm{Si}$ and $\mathrm{Sn} / \mathrm{Na}_{x} \mathrm{Sn}$ interfaces in $\mathrm{Li} / \mathrm{Si}$ and $\mathrm{Na} / \mathrm{Sn}$ systems, respectively ${ }^{[97]}$. (e) Comparison of interfacial microstructures and corresponding residual stress distributions near interface of $\mathrm{Li} / \mathrm{Si}$ and $\mathrm{Na} / \mathrm{Sn}$ systems ${ }^{[97]}$.

\section{Summary and Outlook}

In this brief progress report, we summarize the research history of diffusion kinetics and how this concept can be used to interpret the origin of the electrochemical performances of alloying anodes. The previous theories on diffusion kinetics developed generic criteria of DCR and ICR 
591 diffusion, which is revealed in experiments by the parabolic $\left(L^{2} \propto t\right)$ and linear $(L \propto t)$ rate laws,

592 respectively. Experimental and theoretical studies that aimed to develop high-performance

593 alloying anodes highlighted the importance of diffusion kinetics on the cycle life, rate performance,

594 and practical capacity of anodes. Overall, alloying anodes (e.g., Si, Sb, and P) following ICR

595 commonly show diffusion behaviors, such as anisotropic volume changes, slow diffusion rate, and

596 self-limiting diffusion, which can deteriorate the anode performance during battery cycling. The

597 mechanistic origin of the diffusion kinetics governed by ICR is ascribed to the diffusion of carrier

598 ions by forming a sharp two-phase boundary and abrupt compositional changes across the interface,

599 which develops large residual compressive stresses and impedes further diffusion of carrier ions.

600 Contrary to ICR-governed alloying anodes, those following DCR commonly show isotropic

601 volume changes, a comparatively fast diffusion rate, and reduced self-limiting diffusion, which

602 can improve the anode performance during battery cycling. The criteria to select DCR-governed

603 alloying anodes are based on the interatomic bond strength and yield strength of alloying-anode

604 materials, which can be used as the indicators to assess the ease of diffusion at the interface. When

605 carrier ions diffuse into a low-yielding anode material with a weak interatomic bond strength,

606 carrier-ion diffusion often proceeds by the formation of an amorphous layer and dislocations at the

607 unreacted anode material close to the advancing interface ${ }^{[51]}$. These structural changes can readily

608 relieve residual stresses at the propagating interface and thus facilitate the rapid inflow of carrier

609 ions into the unreacted anode following DCR diffusion. The diffusion behaviors displayed by

610 DCR-governed anodes can also be beneficial in practical applications, of which examples are

611 discussed in the following.

612

613 Reducing the fabrication cost of long-lasting alloying anodes: With regard to the issues 
614 related to relieving residual stresses and improving the cyclability of anodes, some achievements

615 have been made with the design of nanostructure and composite anodes. In light of the size-

616 dependent residual stresses developed in alloying anodes, the use of nanostructured anodes has

617 become a common means by which to prevent anode degradation and improve the cycle life.

618 Nanoscale materials, owing to their large surface-to-volume ratio, can reduce the development of

619 residual stresses ${ }^{[98]}$, which, in turn, can mitigate the reduction of the electrochemical performance

620 arising from ICR diffusion. In addition to nanoscale structures, porous structures can also alleviate

621 the residual stresses caused by large volume changes associated with battery cycling [99-101].

622 Recently, coating and doping of Si and Ge anodes with other elements have also been considered

623 to be effective in controlling volume expansions and associated residual stresses ${ }^{[43,81]}$.

624 Although the above synthesis strategies have greatly improved the cyclability of alloying 625 anodes, there remain critical issues regarding their production costs and complexity. In this respect,

626 the selection of anode materials governed by DCR can provide better battery performance while 627 reducing the additional fabrication cost; when Si and Ge NWs that follow the different diffusion 628 kinetics of ICR and DCR, respectively, are compared, the threshold diameter for the fracture of 629 NWs during lithiation is much larger for Ge $(1200 \mathrm{~nm})^{[72]}$ than that for Si $(300 \mathrm{~nm}){ }^{[71]}$. 630 Furthermore, the critical diameter for full lithiation is also larger for Ge NPs $(\sim 620 \mathrm{~nm}){ }^{[96]}$ than 631 for Si NPs $(\sim 130 \mathrm{~nm})^{[77]}$. These large threshold diameters of Ge allow it to exhibit high mechanical 632 stability and large practical capacity even for anodes with a large size, which requires less 633 fabrication cost than the Si counterparts ${ }^{[95]}$. This suggests that, although the use of nanostructured 634 anodes is effective in improving the cyclability of anodes, the choice of anode materials following 635 DCR diffusion could be another alternative for enhancing the same property. 636 
Improving the rate performance of the conversion-alloying anodes: In addition to the Group IVA and VA elements (e.g., Si, Ge, and $\mathrm{Sn}$ ) discussed in this review, metal oxide anodes $\left(\mathrm{M}_{x} \mathrm{O}_{y}, \mathrm{M}=\mathrm{Zn}, \mathrm{Sn}, \mathrm{Sb}\right.$, etc. $)$ also undergo alloying reactions during battery cycling and have emerged as another important class of anodes for future applications. Some representative examples include $\mathrm{ZnO}$ and $\mathrm{SnO}_{2}$ for Li-ion batteries ${ }^{[102]}$ and $\mathrm{SnO}_{x}$ and $\mathrm{Sb}_{2} \mathrm{O}_{3}$ for $\mathrm{Na}$-ion batteries [17]. The charging and discharging processes of these anodes proceed by following the mechanism known as the "conversion-alloying reaction"; the initial irreversible conversion reaction occurs between $\mathrm{M}_{x} \mathrm{O}_{y}$ and the charge carriers ( $\mathrm{Li}$ or $\mathrm{Na}$ ), and this is followed by the reversible alloying reaction occurring between the reduced $M$ and the charge carriers, according to Eqs. (2) and (3), respectively.

$$
\mathrm{M}_{x} \mathrm{O}_{y}+2 y \mathrm{Li}^{+}+2 y e^{-} \rightarrow x \mathrm{M}+y \mathrm{Li}_{2} \mathrm{O}
$$

$x M+z L i^{+}+z e^{-} \leftrightarrow L i_{z} M_{x}$

In addition to metal oxides, metal sulfides $\left(\mathrm{SnS}_{2}, \mathrm{Sb}_{2} \mathrm{~S}_{3}\right)$ also follow the conversion-alloying mechanism ${ }^{[17,102]}$.

The excellent electrochemical performances of conversion-alloying anodes are attributed to the formation of the $\mathrm{Li}_{2} \mathrm{O}$ (or $\mathrm{Na}_{2} \mathrm{O}$ ) phase in Eq. (2). The major advantage of $\mathrm{Li}_{2} \mathrm{O}$ is that it can mechanically buffer the volumetric expansion during battery cycling and thus improve the cycle life of anodes ${ }^{[102]}$. This is particularly true during the subsequent alloying reaction in Eq. (3). Although the reaction given by Eq. (3) normally causes the volume of the anode to expand by greater than $300 \%{ }^{[48,49]}$, the development of residual stresses in the anodes can be reduced by the presence of the buffer $\mathrm{Li}_{2} \mathrm{O}$ phase, which reduces the energy barrier for diffusion and retards the crack formation and pulverization of the anode materials. Another important feature of the $\mathrm{Li}_{2} \mathrm{O}$ phase is its electrochemical inactivity during battery cycling. According to previous studies on 
660 typical conversion anodes of $\mathrm{TMO}_{x}(\mathrm{TM}=$ transition metal $)$, the reduction or oxidation of $\mathrm{Li}_{2} \mathrm{O}$

661 during battery cycling requires a large amount of energy, which is revealed in voltametric

662 experiments as a large hysteresis loss and thus low energy efficiency of the anodes ${ }^{[103,104] .}$

663 However, unlike the $\mathrm{Li}_{2} \mathrm{O}$ formed in conversion anodes, the same phase formed in conversion-

664 alloying anodes does not react with inflowing/outflowing Li ions, rendering the anode to manifest

665 low-voltage hysteresis and thus high energy efficiency. However, despite the advantages

666 associated with the formation of $\mathrm{Li}_{2} \mathrm{O}$ in conversion-alloying anodes, its poor electric conductivity

667 impedes the diffusion of carrier ions and, in turn, limits the rate performance of the anodes. To

668 solve this problem, research on conversion-alloying anodes hybridized with electrically

669 conductive carbon/graphite is currently underway ${ }^{[105,106]}$.

670 Together with the incorporation of carbonaceous materials, the use of anode materials

671 governed by DCR diffusion can further improve the rate performance of conversion-alloying

672 anodes. As can be seen from the conversion-alloying reactions in Eqs. (2) and (3), the initial

673 conversion reaction is irreversible, whereas the subsequent alloying reaction is reversible during

674 repeated battery cycles. This indicates that the electrochemical performance of these anodes is

675 primarily determined by the alloying reaction, not by the conversion reaction. From this

676 perspective, the concept of the diffusion kinetics of alloying anodes discussed earlier can also be

677 extended to the conversion-alloying anodes. Because alloying anodes following DCR diffusion

678 exhibit faster penetration of the charge carriers into the anodes, conversion-alloying anodes mixed

679 with alloying anodes could display an improved rate performance by taking the advantages of

680 DCR diffusion. There is an increasing number of evidential reports that support this hypothetical

681 concept. Representative examples are $\mathrm{SnO}_{2}{ }^{[107]}$ and $\mathrm{Bi}_{2} \mathrm{O}_{3}{ }^{[108]}$, both of which exhibit long

682 cyclability under fast charging/discharging rates. 
Developing anodes for high-power applications: The two main techniques used to understand the carrier-ion diffusion under high-power operations are the (1) galvanostatic and (2) potentiostatic modes of diffusion experiments. The diffusion of carrier ions under the galvanostatic and potentiostatic modes are controlled by the current density and the electric field (or potential)

688 in the anodes, respectively. From an atomistic perspective, the former operation uses a constant 689 flux of carrier ions in the anode, whereas the latter maintains the constant concentration of the 690 carrier ions at the anode's surface ${ }^{[109]}$. Although the two methods appear to be different from each 691 other, both methods have the same effect in enhancing the diffusion rate of the carrier ions; the 692 greater the current density (or electric field) applied in the anodes, the faster and deeper the carrier 693 ions diffuse into the anodes. This behavior was well observed from the lithiation of Si measured 694 as a function of the current density ${ }^{[92]}$. Experiments have shown that the penetration depths of Li 695 diffusion into $\mathrm{Si}$ increase with increasing current density, while maintaining the preferential 696 diffusion direction along $<110>$ of crystalline $\mathrm{Si}$. This indicates that the practical capacity of the 697 alloying anodes can be increased simply by increasing the current density or the electric field. Although increasing the electric field or the current density is beneficial for improving the 699 capacity of anodes, both methods are ultimately limited by the Li (or $\mathrm{Na}$ ) diffusivity of the 700 electrode materials. When the current rate of the anodes exceeds the threshold current rate (also 701 referred to as the "diffusion-limited C-rate"), the diffusional flux of carrier ions in the anodes is 702 no longer able to keep up with the current rate, resulting in the depletion of carrier ions in the 703 cathodes (anodes) during discharging (charging) ${ }^{[110]}$. This depletion in the charge carriers causes 704 a substantial decrease in the capacity of the battery ${ }^{[111]}$. This phenomenon occurs because the 705 diffusivity of the carrier ions in the electrodes is slower than the current rate applied during high- 
706

707

708

709

710

711

712

713

714

715

716

717

718

719

720 721 anodes suitable for high-power operations.

722

723

724

725

726

727

728

power operations.

The concept of the diffusion kinetics of alloying anodes discussed in this review can be used to develop high-power-density batteries. Owing to their ability to form an amorphous layer and high-density dislocations near the propagating interface (see Figures 7c-d), alloying anodes following DCR diffusion enable the faster diffusion of carrier ions into the anodes. When the diffusivities of alloying anodes are compared, the diffusivities $\left(\sim 3.0 \times 10^{-6} \mathrm{~cm}^{2} \mathrm{~s}^{-1}\right.$ for $\mathrm{Na}-\mathrm{Sn}{ }^{[82]}$ and $\sim 3.6 \times 10^{-7} \mathrm{~cm}^{2} \mathrm{~s}^{-1}$ for $\mathrm{Na}-\mathrm{Ge}^{[112]}$ ) of carrier ions in DCR-governed alloying anodes are greater than those $\left(\sim 5.1 \times 10^{-12} \mathrm{~cm}^{2} \mathrm{~s}^{-1}\right.$ for $\mathrm{Li}-\mathrm{Si}{ }^{[113]}$ and $\sim 3.0 \times 10^{-10} \mathrm{~cm}^{2} \mathrm{~s}^{-1}$ for $\left.\mathrm{Na}-\mathrm{Sb}{ }^{[114]}\right)$ of ICR-governed alloying anodes by more than three to five orders of magnitude. Therefore, the selection of the DCR-governed alloying anodes can prevent the depletion of charge carriers at high current rates, making them suitable for the high-power operations. In addition to the works on improving ionic diffusivity in anodes, additional studies have reported the electric resistivity associated with the occurrence of phase transition during battery cycling is also important in developing anodes for high-power applications ${ }^{[115-117]}$. Further experimental and theoretical studies on the effect of the current rate on diffusion behaviors are required to realize alloying

Although the present review has mainly focused on the diffusion kinetics of alloying anodes, the diffusion behaviors in practical batteries are more complicated and are determined by various external factors. In particular, the electrolyte conditions and associated solid-electrolyte interfaces (SEIs) formed during battery cycles can play an important role in determining the diffusion behaviors of carrier ions. This is especially true when the diffusivity of the carrier ions is lower in the SEIs than in the anodes $[118,119]$. For instance, the carrier-ion diffusivity $\left(\sim 1.8 \times 10^{-13}\right.$ to 
$\left.7297.6 \times 10^{-11} \mathrm{~cm}^{2} \mathrm{~s}^{-1}{ }^{[120]}\right)$ in SEIs is much lower than the typical values (e.g., $\sim 3.0 \times 10^{-6} \mathrm{~cm}^{2} \mathrm{~s}^{-1}$

730 for $\mathrm{Na}-\mathrm{Sn}{ }^{[82]}$ and $\sim 3.6 \times 10^{-7} \mathrm{~cm}^{2} \mathrm{~s}^{-1}$ for $\mathrm{Na}-\mathrm{Ge}^{[112]}$ ) in DCR-governed alloying anodes. In this

731 case, the overall diffusion rate is limited by the SEI, rather than by the propagating interface or the

732 trailing bulk region. Furthermore, the large volumetric expansion of the alloying anodes during

733 lithiation/sodiation can readily destroy the SEI layer formed on the anode surface. All of these

734 behaviors, although lowering the rate performance of the anodes, can alter the diffusion kinetics

735 and associated diffusion behaviors (e.g., anisotropic/isotropic swelling and penetration depth) of

736 the anodes. Therefore, to realize batteries for practical applications, it is essential to optimize the

737 electrolyte conditions and the associated SEIs.

738 Various technical approaches have been devoted to minimizing the detrimental effects of the

739 SEIs on the alloying anodes. The addition of electrolyte additives ${ }^{[121,122]}$ and the coating of the 740 anode with conductive artificial SEIs ${ }^{[123-125]}$ are the effective ways to improve the diffusivity of

741 carrier ions in the SEIs. Other strategies include the enhancement of the structural stability of SEIs

742 dissolving appropriate salts ${ }^{[126]}$ or additives ${ }^{[127,128]}$ in the electrolyte solution and the coating of

743 the anode surface with a conductive and stable carbon layer ${ }^{[129]}$. Although discussions on the

744 electrolyte conditions and SEIs were not covered in detail in this review, the strategies to optimize

745 the electrolyte conditions, in combination with the diffusion kinetics of the alloying anodes, are 746 the key to developing the anodes for future batteries.

748 Acknowledgements

749 This work was supported by the National Research Foundation of Korea (NRF) grant funded by 750 the Korea government (MEST, NRF-2018R1A2B6003927). YSC and DOS are indebted to the 751 Faraday Institution NEXGENNA project (FIRG018) for financial support. 
752

\section{Corresponding Author}

754 E-mail: jclee001@korea.ac.kr (JCL).

755 ORCID

756 Yong-Seok Choi: 0000-0002-3737-2989

757 David O. Scanlon: 0000-0001-9174-8601

758 Jae-Chul Lee: 0000-0002-9294-2163

759 Notes

760 The authors declare no competing financial interest.

761

762 


\section{Biographies}

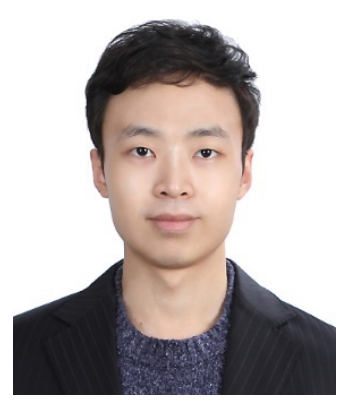

Yong-Seok Choi is a postdoctoral researcher at the Department of Chemistry, University College London. He received his Ph.D. from the Department of Materials Science and Engineering at Korea University in February 2020. He specializes in the application of first-principles techniques for elucidating electronic/atomic structures governing the electrochemical performance of energy storage materials. His experience and interests include electrodes and solid electrolytes for $\mathrm{Na}$-ion batteries.

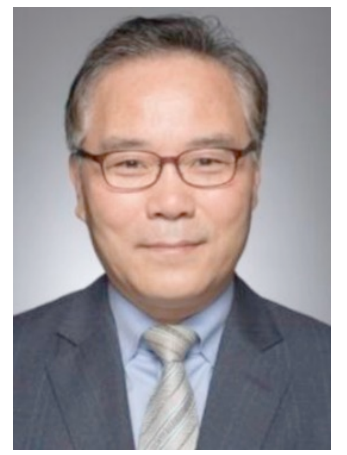

Jae-Chul Lee is a full professor at the College of Engineering, Korea University. He received his B.S. from Korea University, South Korea, in 1985, and his M.S. and Ph.D. in materials science from Michigan State University in 1989 and 1992, respectively. His recent research interests include atomic simulations for elucidating the electric/chemical/mechanical properties of structural materials and batteries.

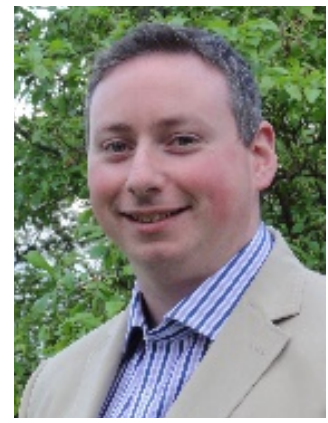

David O. Scanlon is the Chair of Computational Materials Design at the Department of Chemistry at University College London and at Diamond Light Source. He received his B. A. (Mod) in Computational Chemistry and $\mathrm{PhD}$ in Chemistry from Trinity College Dublin, Ireland in 2006 and 2011 respectively. His work is at the forefront of the global effort to explore new materials based on computations and to advance the capacity of first-principles calculations to predict materials properties. 


\section{References}

767 [1] J.-M. Tarascon, M. Armand, in Materials for sustainable energy: a collection of peer-

768 reviewed research and review articles from Nature Publishing Group, World Scientific, Republic 769 of Singapore $2011,171$.

770 [2] N. Nitta, F. Wu, J. T. Lee, G. Yushin, Mater. Today 2015, 18, 252.

771 [3] P. K. Nayak, E. M. Erickson, F. Schipper, T. R. Penki, N. Munichandraiah, P. Adelhelm, 772 H. Sclar, F. Amalraj, B. Markovsky, D. Aurbach, Adv. Energy Mater. 2018, 8, 1702397.

773 [4] C. Capasso, O. Veneri, Appl. Energy 2014, 136, 921.

774 [5] P. Gibot, M. Casas-Cabanas, L. Laffont, S. Levasseur, P. Carlach, S. Hamelet, J.-M. 775 Tarascon, C. Masquelier, Nat. Mater. 2008, 7, 741.

776 [6] M. Noel, V. Suryanarayanan, J. Power Sources 2002, 111, 193.

777 [7] J. Xu, F. Lin, M. M. Doeff, W. Tong, J. Mater. Chem. A 2017, 5, 874.

778 [8] P. Rozier, J. M. Tarascon, J. Electrochem. Soc. 2015, 162, A2490.

779 [9] L. Zhang, M. A. Reddy, X.-M. Lin, Z. Zhao-Karger, M. Fichtner, J. Alloys Compd. 2017, $780 \quad 724,1101$.

$781 \quad$ [10] Y. Lu, L. Yu, X. W. D. Lou, Chem 2018, 4, 972.

782 [11] H. Yin, Q. Li, M. Cao, W. Zhang, H. Zhao, C. Li, K. Huo, M. Zhu, Nano Res. 2017, 10, 7832156.

784 [12] A. M. Chockla, K. C. Klavetter, C. B. Mullins, B. A. Korgel, ACS Appl. Mater. Inter. 2012, $7854,4658$.

786 [13] J. Qian, D. Qiao, X. Ai, Y. Cao, H. Yang, Chem. Commun. 2012, 48, 8931.

787 [14] C.-M. Park, S. Yoon, S.-I. Lee, J.-H. Kim, J.-H. Jung, H.-J. Sohn, J. Electrochem. Soc. $788 \quad 2007,154$, A917. 
[15] Y. Yao, M. T. McDowell, I. Ryu, H. Wu, N. Liu, L. Hu, W. D. Nix, Y. Cui, Nano Lett. 2011, 11, 2949.

791 [16] W. M. Zhang, J. S. Hu, Y. G. Guo, S. F. Zheng, L. S. Zhong, W. G. Song, L. J. Wan, Adv. 792 Mater. 2008, 20, 1160.

793 [17] H. Zhang, I. Hasa, S. Passerini, Adv. Energy Mater. 2018, 8, 1702582.

794 [18] M. J. Chon, V. A. Sethuraman, A. McCormick, V. Srinivasan, P. R. Guduru, Phys. Rev. 795 Lett. 2011, 107, 045503.

796 [19] X. H. Liu, H. Zheng, L. Zhong, S. Huang, K. Karki, L. Q. Zhang, Y. Liu, A. Kushima, W. 797 T. Liang, J. W. Wang, Nano Lett. 2011, 11, 3312.

798 [20] Z. Cui, F. Gao, J. Qu, J. Mech. Phys. Solids 2013, 61, 293.

799 [21] H. Schmalzried, Chemical kinetics of solids, John Wiley \& Sons, Germany 2008.

800 [22] D. J. Young, High temperature oxidation and corrosion of metals, Vol. 1, Elsevier, $801 \quad$ Netherlands 2008.

802 [23] H. Schmalzried, Solid-State Reactions between Oxides in Defects and transport in oxides, 803 Springer, Germany 1974, 83.

804 [24] M. H. Davies, C. E. Birchenall, JOM 1951, 3, 877.

805 [25] V. B. Voitovich, V. A. Lavrenko, V. M. Adejev, E. I. Golovko, Oxid. Met. 1995, 43, 509.

806 [26] H. I. Aaronson, T. Abe, J. Bradley, W. A. Clark, T. Obara, Mater. Sci. 1979.

807 [27] P. W. Bach, Vol. 13, Technische Hogeschool Twente 1981, 1.

808 [28] R. M. Cannon, W. H. Rhodes, A. H. Heuer, J. Am. Ceram. Soc. 1980, 63, 46.

809 [29] P. Murray, G. F. Carey, J. Comput. Phys. 1988, 74, 440.

810 [30] P. Murray, G. F. Carey, J. Appl. Phys. 1989, 65, 3667.

811 [31] G. P. Krielaart, J. Sietsma, S. Van Der Zwaag, Mater. Sci. Eng. A 1997, 237, 216. 
812 [32] C. R. Gorla, W. E. Mayo, S. Liang, Y. Lu, J. Appl. Phys. 2000, 87, 3736.

813 [33] C. Korte, N. Ravishankar, C. B. Carter, H. Schmalzried, Solid State Ion. 2002, 148, 111.

814 [34] J. K. Farrer, C. B. Carter, J. Mater. Sci. 2006, 41, 5169.

815 [35] T. Akashi, M. Nanko, T. Maruyama, Y. Shiraishi, J. Tanabe, J. Electrochem. Soc. 1998, $816145,2090$.

817 [36] J. Y. Huang, L. Zhong, C. M. Wang, J. P. Sullivan, W. Xu, L. Q. Zhang, S. X. Mao, N. S. 818 Hudak, X. H. Liu, A. Subramanian, Science 2010, 330, 1515.

819 [37] S. W. Lee, M. T. McDowell, J. W. Choi, Y. Cui, Nano Lett. 2011, 11, 3034.

820 [38] A. F. Bower, P. R. Guduru, V. A. Sethuraman, J. Mech. Phys. Solids 2011, 59, 804.

821 [39] Z. Cui, F. Gao, J. Qu, J. Mech. Phys. Solids 2012, 60, 1280.

822 [40] K. Zhao, M. Pharr, Q. Wan, W. L. Wang, E. Kaxiras, J. J. Vlassak, Z. Suo, J. Electrochem. 823 Soc. 2011, 159, A238.

824 [41] J.-H. Seo, C.-Y. Chou, Y.-H. Tsai, Y. Cho, T.-Y. Seong, W.-J. Lee, M.-H. Cho, J.-P. Ahn, 825 G. S. Hwang, I.-S. Choi, RSC Adv. 2015, 5, 17438.

826 [42] X. H. Liu, J. W. Wang, S. Huang, F. Fan, X. Huang, Y. Liu, S. Krylyuk, J. Yoo, S. A. 827 Dayeh, A. V. Davydov, Nat. Nanotech. 2012, 7, 749.

828 [43] X. H. Liu, L. Q. Zhang, L. Zhong, Y. Liu, H. Zheng, J. W. Wang, J.-H. Cho, S. A. Dayeh, 829 S. T. Picraux, J. P. Sullivan, Nano Lett. 2011, 11, 2251.

830 [44] J.-H. Park, Y.-S. Choi, Y.-W. Byeon, J.-P. Ahn, J.-C. Lee, Nano Energy 2019, 65, 104041. 831 [45] X. H. Liu, S. Huang, S. T. Picraux, J. Li, T. Zhu, J. Y. Huang, Nano Lett. 2011, $11,3991$. 832 [46] X. Lu, E. R. Adkins, Y. He, L. Zhong, L. Luo, S. X. Mao, C.-M. Wang, B. A. Korgel, 833 Chem. Mater. 2016, 28, 1236.

834 [47] E. W. Haycock, J. Electrochem. Soc. 1959, 106, 771. 
835 [48] J. Woods, N. Bhattarai, P. Chapagain, Y. Yang, S. Neupane, Nano Energy 2019, 56, 619.

836 [49] M. Lao, Y. Zhang, W. Luo, Q. Yan, W. Sun, S. X. Dou, Adv. Mater. 2017, 29, 1700622.

837 [50] M. T. McDowell, I. Ryu, S. W. Lee, C. Wang, W. D. Nix, Y. Cui, Adv. Mater. 2012, 24, $838 \quad 6034$.

839 [51] Y.-S. Choi, J.-H. Park, J.-P. Ahn, J.-C. Lee, Chem. Mater. 2019, 31, 1696.

840 [52] H. Mehrer, Diffusion in solids: fundamentals, methods, materials, diffusion-controlled 841 processes, Vol. 155, Springer Science \& Business Media, United States 2007.

842 [53] H. Yang, S. Huang, X. Huang, F. Fan, W. Liang, X. H. Liu, L.-Q. Chen, J. Y. Huang, J. 843 Li, T. Zhu, Nano Lett. 2012, 12, 1953.

844 [54] H. Jung, M. Lee, B. C. Yeo, K.-R. Lee, S. S. Han, J. Phys. Chem. C 2015, 119, 3447.

845 [55] A. Ostadhossein, E. D. Cubuk, G. A. Tritsaris, E. Kaxiras, S. Zhang, A. C. T. Van Duin, 846 Phys. Chem. Chem. Phys. 2015, 17, 3832.

847 [56] J. Rohrer, A. Moradabadi, K. Albe, P. Kaghazchi, J. Power Sources 2015, 293, 221.

848 [57] M. K. Y. Chan, C. Wolverton, J. P. Greeley, J. Am. Chem. Soc. 2012, 134, 14362.

849 [58] H. Tachikawa, Y. Nagoya, T. Fukuzumi, J. Power Sources 2010, 195, 6148.

850 [59] H. Tachikawa, J. Phys. Chem. C 2007, 111, 13087.

851 [60] H. Tachikawa, A. Shimizu, J. Phys. Chem. B 2005, 109, 13255.

852 [61] Y.-S. Choi, J.-H. Park, J.-P. Ahn, J.-C. Lee, Sci.Rep. 2017, 7, 1.

853 [62] W. Xia, Q. Zhang, F. Xu, H. Ma, J. Chen, K. Qasim, B. Ge, C. Zhu, L. Sun, J. Phys. Chem. $854 \quad C$ 2016, $120,5861$.

855 [63] J. Sun, H.-W. Lee, M. Pasta, H. Yuan, G. Zheng, Y. Sun, Y. Li, Y. Cui, Nat. Nanotech. $856 \mathbf{2 0 1 5}, 10,980$.

857 [64] T. Chen, P. Zhao, X. Guo, S. Zhang, Nano Lett. 2017, 17, 2299. 
858 [65] Y.-W. Byeon, Y.-S. Choi, J.-P. Ahn, J.-C. Lee, ACS Appl. Mater. Inter. 2018, 10, 41389.

859 [66] B. Schwartz, J. Electrochem. Soc. 1967, 114, 285.

860 [67] R. Leancu, N. Moldovan, L. Csepregi, W. Lang, Sens. Actuators A 1995, 46, 35.

861 [68] Y.-S. Choi, Y.-W. Byeon, J.-P. Ahn, J.-C. Lee, Nano Lett. 2017, 17, 679.

862 [69] B.-H. Kim, M. Ariesto Pamungkas, M. Park, G. Kim, K.-R. Lee, Y.-C. Chung, Appl. Phys. 863 Lett. 2011, 99, 143115.

864 [70] T. Kennedy, M. Brandon, K. M. Ryan, Adv. Mater. 2016, 28, 5696.

865 [71] I. Ryu, J. W. Choi, Y. Cui, W. D. Nix, J. Mech. Phys. Solids 2011, 59, 1717.

866 [72] S. W. Lee, I. Ryu, W. D. Nix, Y. Cui, Extreme Mech. Lett. 2015, 2, 15.

867 [73] M. Sun, K. Qi, X. Li, Q. Huang, J. Wei, Z. Xu, W. Wang, X. Bai, ChemElectroChem 2016, $8683,1296$.

869 [74] M. T. McDowell, S. W. Lee, I. Ryu, H. Wu, W. D. Nix, J. W. Choi, Y. Cui, Nano Lett. $870 \quad 2011,11,4018$.

871 [75] M. T. McDowell, S. W. Lee, J. T. Harris, B. A. Korgel, C. Wang, W. D. Nix, Y. Cui, Nano $872 \quad$ Lett. 2013, 13, 758.

873 [76] J. W. Wang, Y. He, F. Fan, X. H. Liu, S. Xia, Y. Liu, C. T. Harris, H. Li, J. Y. Huang, S. 874 X. Mao, Nano Lett. 2013, 13, 709.

875 [77] X. H. Liu, F. Fan, H. Yang, S. Zhang, J. Y. Huang, T. Zhu, ACS Nano 2013, 7, 1495.

876 [78] A. D. Drozdov, P. Sommer-Larsen, J. deClaville Christiansen, J. Appl. Phys. 2013, 114, 877223514.

878 [79] T. Shimura, M. Shimizu, S. Horiuchi, H. Watanabe, K. Yasutake, M. Umeno, Appl. Phys. 879 Lett. 2006, 89, 111923. 
880 [80] K.-W. Park, C.-M. Lee, M. Wakeda, Y. Shibutani, M. L. Falk, J.-C. Lee, Acta Mater. 2008, $881 \quad 56,5440$.

882 [81] L. C. Loaiza, L. Monconduit, V. Seznec, Small 2020, 16, 1905260.

883 [82] Y.-S. Choi, Y.-W. Byeon, J.-H. Park, J.-H. Seo, J.-P. Ahn, J.-C. Lee, ACS Appl. Mater. 884 Inter. 2018, 10, 560 .

885 [83] A. Nie, L.-Y. Gan, Y. Cheng, H. Asayesh-Ardakani, Q. Li, C. Dong, R. Tao, F. Mashayek, 886 H.-T. Wang, U. Schwingenschlögl, ACS Nano 2013, 7, 6203.

887 [84] M. Legros, G. Dehm, E. Arzt, T. J. Balk, Science 2008, 319, 1646.

888 [85] H. Asayesh-Ardakani, W. Yao, Y. Yuan, A. Nie, K. Amine, J. Lu, R. Shahbazian-Yassar, 889 Small Methods 2017, 1, 1700202.

890 [86] K. E. Gregorczyk, Y. Liu, J. P. Sullivan, G. W. Rubloff, ACS Nano 2013, 7, 6354.

891 [87] G. R. Love, Acta Metall. 1964, 12, 731.

892 [88] J. Huang, M. Meyer, V. Pontikis, Phys. Rev. Lett. 1989, 63, 628.

893 [89] J. Rabier, M. P. Puls, Philos. Mag. A 1989, 59, 533.

894 [90] P. Shewmon, Diffusion in solids, Springer, Switzerland 2016.

895 [91] S. Zhang, npj Comput. Mater. 2017, 3, 1.

896 [92] J. L. Goldman, B. R. Long, A. A. Gewirth, R. G. Nuzzo, Adv. Funct. Mater. 2011, 21, 8972412.

898 [93] S. Mirabella, D. De Salvador, E. Napolitani, E. Bruno, F. Priolo, J. Appl. Phys. 2013, 113, 8993.

900 [94] C. K. Chan, H. Peng, G. Liu, K. McIlwrath, X. F. Zhang, R. A. Huggins, Y. Cui, Nat. $901 \quad$ Nanotech. 2008, 3, 31 . 
902 [95] X. H. Liu, Y. Liu, A. Kushima, S. Zhang, T. Zhu, J. Li, J. Y. Huang, Adv. Energy Mater. $903 \mathbf{2 0 1 2}, 2,722$.

904 [96] W. Liang, H. Yang, F. Fan, Y. Liu, X. H. Liu, J. Y. Huang, T. Zhu, S. Zhang, ACS Nano $905 \mathbf{2 0 1 3}, 7,3427$.

906 [97] Y.-W. Byeon, J.-P. Ahn, J.-C. Lee, Small 2020, in print.

907 [98] C. K. Chan, R. N. Patel, M. J. O’connell, B. A. Korgel, Y. Cui, ACS Nano 2010, 4, 1443.

908 [99] Q. Xiao, M. Gu, H. Yang, B. Li, C. Zhang, Y. Liu, F. Liu, F. Dai, L. Yang, Z. Liu, Nat. 909 Commun. 2015, 6, 1.

910 [100] J. Cho, J. Mater. Chem. 2010, 20, 4009.

911 [101] Z. Jia, T. Li, J. Power Sources 2015, 275, 866.

912 [102] D. Bresser, S. Passerini, B. Scrosati, Energy Environ. Sci. 2016, 9, 3348.

913 [103] N. Nitta, G. Yushin, Part. Part. Syst. Charact. 2014, 31, 317.

914 [104] M. R. Palacin, Chem. Soc. Rev. 2009, 38, 2565.

915 [105] M.-G. Park, G.-K. Sung, N.-E. Sung, J.-H. Kim, C.-M. Park, J. Power Sources 2016, 328, 916607.

917 [106] S. Böhme, K. Edström, L. Nyholm, Electrochim. Acta 2015, 179, 482.

918 [107] P. Lian, X. Zhu, S. Liang, Z. Li, W. Yang, H. Wang, Electrochim. Acta 2011, 56, 4532.

919 [108] M.-K. Kim, S.-H. Yu, A. Jin, J. Kim, I.-H. Ko, K.-S. Lee, J. Mun, Y.-E. Sung, Chem. 920 Commun. 2016, 52, 11775.

921 [109] Y.-T. Cheng, M. W. Verbrugge, J. Power Sources 2009, 190, 453.

922 [110] C. Heubner, M. Schneider, A. Michaelis, Adv. Energy Mater. 2020, 10, 1902523.

923 [111] B. Byles, N. Palapati, A. Subramanian, E. Pomerantseva, APL Mater. 2016, 4, 046108.

924 [112] G. Pekar, A. Singaevsky, Mater. Sci. Semicond. Process. 2017, 64, 10. 
925 [113] N. Ding, J. Xu, Y. Yao, G. Wegner, X. Fang, C. Chen, I. Lieberwirth, Solid State Ion. 2009, $926180,222$.

927 [114] L. Baggetto, P. Ganesh, C.-N. Sun, R. A. Meisner, T. A. Zawodzinski, G. M. Veith, J. 928 Mater. Chem. A 2013, 1, 7985.

929 [115] Y.-S. Choi, Y.-W. Byeon, J.-P. Ahn, J.-C. Lee, J. Mater. Chem. A 2018, 6, 9428.

930 [116] V. Viswanathan, J. Nørskov, A. Speidel, R. Scheffler, S. Gowda, A. Luntz, J. Phys. Chem. 931 Lett. 2013, 4, 556.

932 [117] Y.-S. Choi, J.-C. Lee, J. Mater. Chem. A 2018, 6, 11531.

933 [118] P. Biswal, S. Stalin, A. Kludze, S. Choudhury, L. A. Archer, Nano Lett. 2019, 19, 8191.

934 [119] Y. He, X. Ren, Y. Xu, M. H. Engelhard, X. Li, J. Xiao, J. Liu, J.-G. Zhang, W. Xu, C. 935 Wang, Nat. Nanotech. 2019, 14, 1042.

936 [120] L. Benitez, J. M. Seminario, J. Electrochem. Soc. 2017, 164, E3159.

937 [121] J. Qian, W. A. Henderson, W. Xu, P. Bhattacharya, M. Engelhard, O. Borodin, J.-G. Zhang, 938 Nat. Commun. 2015, 6, 1.

939 [122] M. D. Tikekar, S. Choudhury, Z. Tu, L. A. Archer, Nat. Energy 2016, 1, 1.

940 [123] X. Wang, L. Fan, D. Gong, J. Zhu, Q. Zhang, B. Lu, Adv. Funct. Mater. 2016, $26,1104$.

941 [124] W. Liu, P. Liu, D. Mitlin, Adv. Energy Mater. 2020, 10, 2002297.

942 [125] J.-H. Park, Y.-S. Choi, H.-J. Lee, H.-C. Shim, J.-P. Ahn, J.-C. Lee, Nano Lett. 2019, 19, 9433692.

944 [126] G. Singh, B. Acebedo, M. C. Cabanas, D. Shanmukaraj, M. Armand, T. Rojo, Electrochem. 945 Commun. 2013, 37, 61.

946 [127] K. Kubota, S. Komaba, J. Electrochem. Soc. 2015, 162, A2538. 
947 [128] S. Komaba, T. Ishikawa, N. Yabuuchi, W. Murata, A. Ito, Y. Ohsawa, ACS Appl. Mater.

$948 \quad$ Inter. 2011, 3, 4165.

949 [129] S. Menkin, D. Golodnitsky, E. Peled, Electrochem. Commun. 2009, 11, 1789. 